\title{
Lower semicontinuity for the Helfrich problem
}

\section{Sascha Eichmann ${ }^{1}$}

Received: 3 September 2019 / Accepted: 20 May 2020 / Published online: 15 June 2020

(c) The Author(s) 2020

\begin{abstract}
We minimise the Canham-Helfrich energy in the class of closed immersions with prescribed genus, surface area, and enclosed volume. Compactness is achieved in the class of oriented varifolds. The main result is a lower-semicontinuity estimate for the minimising sequence, which is in general false under varifold convergence by a counter example by Große-Brauckmann. The main argument involved is showing partial regularity of the limit. It entails comparing the Helfrich energy of the minimising sequence locally to that of a biharmonic graph. This idea is by Simon, but it cannot be directly applied, since the area and enclosed volume of the graph may differ. By an idea of Schygulla we adjust these quantities by using a two parameter diffeomorphism of $\mathbb{R}^{3}$.
\end{abstract}

Keywords Closed immersions · Canham-Helfrich energy · Lower semicontinuity · Variational problem $\cdot$ Oriented varifolds

Mathematics Subject Classification $26 \mathrm{~B} 15 \cdot 35 \mathrm{~J} 35 \cdot 49 \mathrm{~J} 45 \cdot 49 \mathrm{Q} 20 \cdot 58 \mathrm{~J} 90$

\section{Introduction}

This article deals with minimising the Helfrich energy for closed oriented smooth connected two-dimensional immersions $f: \Sigma \rightarrow \mathbb{R}^{3}$, which is defined as

$$
W_{H_{0}}(f):=\int_{\Sigma}\left(\bar{H}-H_{0}\right)^{2} \mathrm{~d} \mu_{g} .
$$

Here $\bar{H}$ is the scalar mean curvature, i.e. the sum of the principal curvatures with respect to a choosen unit normal of $f$. We call $\mu_{g}$ the area measure on $\Sigma$ induced by $f$ and the euclidean metric of $\mathbb{R}^{3}$. Furthermore, $H_{0} \in \mathbb{R}$ is called spontaneous curvature. This energy was introduced by Helfrich [20] and Canham [3] to model the shape of blood cells. Hence it is

The author thanks Prof. Reiner Schätzle for discussing the Helfrich energy and providing insight into geometric measure theory.

Sascha Eichmann

sascha.eichmann@math.uni-tuebingen.de

1 Mathematisch-Naturwissenschaftliche Fakultät, Eberhard Karls Universität Tübingen, Auf der Morgenstelle 10, 72076 Tübingen, Germany 
called the Canham-Helfrich or short Helfrich energy. We recover the Willmore energy by setting $H_{0}=0$ and multiplying by $\frac{1}{4}$, i.e.

$$
W_{\text {Will }}(f):=\frac{1}{4} \int_{\Sigma}|\bar{H}|^{2} d A=\frac{1}{4} W_{0}(f) .
$$

The Willmore energy goes back to Thomsen [40]. He denoted critical points of the Willmore energy as conformal minimal surfaces. Willmore later revived the mathematical discussion in [41]. Please note, that for the Willmore energy an orientation is not needed, contrary to the Helfrich energy. Hence we need a fixated normal. We assume $\Sigma$ to have a continuous orientation $\tau$, given as a 2 -form. Then we set $v_{f}:=*(d f(\tau)) \in \partial B_{1}(0) \subset \mathbb{R}^{3}$ as the unit normal of $f$. Here $*$ denotes the Hodge-*-Operator. Furthermore, we like to prescribe the area and enclosed volume of $f$. Therefore we set

$$
\operatorname{Area}(f):=\int_{\Sigma} \mathrm{d} \mu_{g}, \quad \operatorname{Vol}(f):=\frac{1}{3} \int_{\Sigma} f(x) \cdot v_{f}(x) \mathrm{d} \mu_{g}(x) .
$$

Please note that, if $f$ is an embedding and $v_{f}$ the outer normal, Vol $(f)$ would be the volume of the set enclosed by $f$. In the general case, $\operatorname{Vol}(f)$ may become negativ dependend on the orientation.

Minimisers of such a problem satisfy the following Euler-Lagrange equation (see, e.g. [31, Eq. (31)])

$$
2 \Delta_{f} \bar{H}+4 \bar{H}\left(\frac{1}{4} \bar{H}^{2}-K\right)-2 H_{0} K-H_{0}^{2} \bar{H}-\lambda_{A} \bar{H}+\lambda_{V}=0 .
$$

Here, $\lambda_{A} \in \mathbb{R}$ and $\lambda_{V} \in \mathbb{R}$ correspond to Lagrange multipliers for the prescribed area and enclosed volume, respectively. This differential equation is highly nonlinear since the Laplace-Beltrami $\Delta_{f}$ depends on the unknown immersion $f$. Furthermore, it is of fourth order, hence standard techniques like the maximum principle are not applicable. Nevertheless a lot of important results concerning such problems have been achieved: Existence of closed Willmore surfaces of arbitrary genus has been shown in the papers [39] and [2]. If the ambient space becomes a general 3-manifold, the problem has been examined in, e.g. [30].

Prescribing additional conditions and showing existence has also been very successful, i.e. the isoperimetric ratio in [23] and [36], the area in [25], boundary conditions in [6, 32, 35] and [9]. Finally, the Willmore conjecture was shown to be true in [28]. Further research and references for Willmore problems are summarized in the surveys [21] rsp. [19]. The class of axisymmetric surfaces is especially important for modelling purposes; see, e.g. [11]. Also some existence results can be more readily achieved in this class, see, e.g. [7, 12] or [14] and solutions to (1.4) can be analysed in greater depths, e.g. the behaviour of singularities in [16].

Furthermore, the addition of the orientation complicates and changes the situation in the Helfrich setting. For example, the class of invariances is notably smaller (see, e.g. [8]) and lower semi-continuity is in general false under varifold convergence; see [18, p. 550, Remark (ii)] for a counterexample. Nevertheless, some progress has been made: Existence of Helfrich surfaces with prescribed surface area near the sphere has been achieved in [24] by examining the corresponding $L^{2}$-flow. This result has been extendend to a general existence result for spherical Helfrich immersions with prescribed area and enclosed volume in [29] by variational parametric methods. The axisymmetric case has been handled independently in [5] and [4]. A more general approach was used in [10] by working with 
Gauss-graphs. There lower semicontinuity was shown, but the limit has to be in $C^{2}$, which is a priori not clear.

In general, this is a hard problem, since the Helfrich energy for oriented varifolds lacks a variational characterisation (cf. (2.3) and cf. [13, p. 3]).

In this paper, we will show a lower-semicontinuity estimate for minimising sequences with an arbitrary but fixed topology by an ambient approach, i.e. geometric measure theory. In the case of spherical topology this has been achieved with a parametric approach in [29, Thm. 3.3]. The case of embeddings still remains open, since we do not have a Li-Yau type inequality. This prevents our arguments to be adaptable to this situation as explained in Remark 5.2.

For our minimising procedure, we introduce the following set for a given two-dimensional smooth connected manifold $\Sigma$ without boundary, which fixes the topology.

$$
M_{\text {Area }_{0}, \operatorname{Vol}_{0}}:=\left\{\begin{array}{c}
f: \Sigma \rightarrow \\
\mathbb{R}^{3}, 2 \text {-dimensional oriented smooth immersion, } \\
\operatorname{Area}(\Sigma)=\operatorname{Area}_{0}, \operatorname{Vol}(\Sigma)=\operatorname{Vol}_{0}, 0 \in f(\Sigma)
\end{array}\right\}
$$

for a positive parameter $\mathrm{Area}_{0}>0$ and a nontrivial real parameter $\operatorname{Vol}_{0} \in \mathbb{R} \backslash\{0\}$. Furthermore let us call

$$
E_{H_{0}, \text { Area }_{0}, \text { Vol }_{0}}:=\inf \left\{W_{H_{0}}(f), f \in M_{\text {Area }_{0}, \text { Vol }_{0}}\right\} .
$$

Now we can state our main result in case $M_{\text {Area }_{0}, V_{0} l_{0}} \neq \emptyset$ (For unknown terminology please consult Sect. 2):

Theorem 1.1 Let $f_{k} \in M_{\text {Area }_{0}, V_{0} l_{0}}$ be a minimising sequence, i.e. it satisfies $W_{H_{0}}\left(f_{k}\right) \rightarrow E_{H_{0}, \text { Area }_{0}, \text { Vol }_{0}}$. Let also $V_{k}^{0}$ be the sequence of oriented integral 2 varifolds corresponding to $f_{k}$ by (2.4). Then there exists an oriented integral 2 varifold $V^{0}$ on $\mathbb{R}^{3}$ and a subsequence $V_{k_{j}}^{0}$, such that

$$
\begin{aligned}
& V_{k_{j}} \rightarrow V^{0} \text { as oriented varifolds, } \\
& \operatorname{Area}\left(V^{0}\right)=\operatorname{Area}_{0}, \quad \operatorname{Vol}\left(V^{0}\right)=\operatorname{Vol}_{0} \neq 0 .
\end{aligned}
$$

More importantly, this subsequence enjoys a lower-semicontinuity estimate:

$$
W_{H_{0}}\left(V^{0}\right) \leq \liminf _{j \rightarrow \infty} V_{k_{j}}^{0} .
$$

Furthermore, there exist at most finitely many bad points, such that $V^{0}$ is locally a union of $C^{1, \beta} \cap W^{2,2}$ graphs outside these bad points. For a precise statement of the graphical decomposition please refer to Lemma 5.1 .

The proof works in two major steps. The first is to show some preliminary regularity of $V^{0}$ (see Lemma 5.1). After this the lower-semicontinuity estimate follows precisely as in [13, Lemma 4.1], hence we will not include this step here.

The initial regularity is shown by comparing the Helfrich energy of the minimising sequence to a biharmonic replacement. This idea was first used by Simon in [39]. Since the biharmonic replacement does not have the same area and/or enclosed volume, we will correct these parameters by an idea of Schygulla [36]. We generalise Schygulla's techniques to the case of immersions and to two prescribed quantities. This idea essentially is adjusting these quantities of the biharmonic replacement outside of the replacement region by a two parameter diffeomorphism. 
The paper is build up as follows: Sect. 2 is concerned with compactness in the class of oriented varifolds. Here the Helfrich energy, the area and the enclosed volume are formulated for these varifolds. Next in Sect. 3 we examine our definition of enclosed volume for oriented varifolds in greater detail and for example calculate an Euler-Lagrange equation. Afterwards we construct the aforementioned two parameter diffeomorphism and analyse these in 4 . In Sect. 5 we finally show the initial $C^{1, \beta}$-regularity, from which the lower-semicontinuity estimate follows. In Appendix A, we collect some usefull results for our reasoning.

\section{Compactness}

In this chapter, we recall the necessary results and objects to obtain compactness for a minimising sequence in measure theoretic terms:

Since the Helfrich energy depends on the orientation, we will work with oriented varifolds in our variational framework. Oriented varifolds were introduced by Hutchinson in [22, Sect. 3]. We recall the necessary definitions here (see also [13, Appendix B]). First

$$
G^{0}(2,3)=\left\{\tau^{1} \wedge \tau^{2} \in \Lambda_{2} \mathbb{R}^{3}: \tau^{1}, \tau^{2} \in \mathbb{R}^{3},\left|\tau^{1}\right|=\left|\tau^{2}\right|=1, \tau^{1} \perp \tau^{2}\right\}
$$

is called the oriented Grassmannian manifold of two-dimensional oriented linear subspaces in $\mathbb{R}^{3}$. Since we need to connect orientations with normals, we also need the Hodge star operator $*: G^{0}(2,3) \rightarrow \partial B_{1}(0)$. In our setting, $*$ becomes just the cross product, i.e. $*\left(\tau^{1} \wedge \tau^{2}\right)=\tau^{1} \times \tau^{2}$. An oriented integral 2-varifold on an open set $\Omega \subset \mathbb{R}^{3}$ is given by a countable 2-rectifiable set $M \subset O, \mathcal{H}^{2}$-measurable densities $\theta_{+}, \theta_{-}: M \rightarrow \mathbb{N}_{0}$ and an orientation $\xi: M \rightarrow G^{0}(2,3)$, such that $*(\xi(x)) \perp T_{x} M$ for $\mathcal{H}^{2}$ a.e. $x \in M$. Then the corresponding oriented varifold is a Radon measure on $\Omega \times G^{0}(2,3)$ given for $\Phi \in C_{0}^{0}\left(\Omega \times G^{0}(2,3)\right)$ by

$$
V^{0}\left[M, \theta_{+}, \theta_{-}, \xi\right](\Phi):=\int_{M} \Phi(x, \xi(x)) \theta_{+}(x)+\Phi(x,-\xi(x)) \theta_{-} \mathrm{d} \mathcal{H}^{2}(x)
$$

Furthermore, we need to define the Helfrich energy for such an oriented integral varifold. Hence we need to make sense of a mean curvature. For this, let $\pi^{0}: \mathbb{R}^{3} \times G^{0}(2,3)$ be given by $\pi^{0}(x, \xi)=x$. Then the mass of $V^{0}$ is defined as

$$
\mu_{V^{0}}:=\pi^{0}\left(V^{0}\right)=\left(\theta_{+}+\theta_{-}\right) \mathcal{H}^{2}\lfloor M,
$$

which is also an integral varifold in the sense of [38, Sect. 15]. The first variation of $V^{0}$ is defined as the first variation of $\mu_{V^{0}}$, i.e. $\delta V^{0}:=\delta \mu_{V^{0}}$ Thus we define the mean curvature vector of $V^{0}$ to be the mean curvature vector of $\mu_{V^{0}}$ (cf. [38, Sect. 16]). We say $V^{0}$ has a mean curvature vector $H_{V^{0}} \in L^{2}\left(\mu_{V^{0}}\right)$, if $H_{V^{0}}$ is square integrable w.r.t. $\mu_{V^{0}}$ and for every $X \in C_{0}^{1}\left(\Omega, \mathbb{R}^{3}\right)$ we have

$$
\delta V^{0}(X)=-\int_{\Omega} H_{V^{0}} \cdot X \mathrm{~d} \mu_{V^{0}}
$$

This means that $\mu_{V^{0}}$ does not have a generalized boundary in the sense of [38, Sect. 39].

The integral 2 current associated with $V^{0}$ is given by 


$$
\left[\left|V^{0}\right|\right](\omega):=\int_{G^{0}(\Omega)}\langle\omega(x), \xi\rangle \mathrm{d} V^{0}(x, \xi), \omega \in C_{0}^{\infty}\left(\Omega, \Lambda^{2} \mathbb{R}^{3}\right) .
$$

We choose the same notations for currents as in [38, Chapter 6].

For an integral oriented varifolds $V^{0}=V^{0}\left[M, \theta_{+}, \theta_{-}, \xi\right]$ the current satisfies

$$
\left[\left|V^{0}\right|\right](\omega)=\int_{M}\langle\omega(x), \xi(x)\rangle\left(\theta_{+}(x)-\theta_{-}(x)\right) \mathrm{d} \mathcal{H}^{2}
$$

and is therefore integral as well. We will call $\partial\left[\left|V^{0}\right|\right]$ the boundary in the sense of currents of $V^{0}$. Here $\partial$ is the boundary operator for currents (see, e.g. [38, Eq. (26.3)]).

Convergence of oriented varifolds is defined as weak convergence of the corresponding Radon measures. That is, we say a sequence of oriented integral varifolds $V_{k}^{0}$ on $\Omega \subset \mathbb{R}^{3}$ converges weakly to an oriented integral varifold $V^{0}$, iff for all $\Phi \in C_{0}^{0}\left(\Omega \times G^{0}(2,3)\right)$ we have $V_{k}^{0}(\Phi) \rightarrow V^{0}(\Phi)$. Then [22, Theorem 3.1] gives us, that the following set is sequentially compact with respect to oriented varifold convergence:

$$
\begin{gathered}
\left\{V^{0} \text { oriented integral varifold : } \forall \Omega^{\prime} \subset \subset \Omega \exists C\left(\Omega^{\prime}\right)<\infty:\right. \\
\left.\mu_{V^{0}}\left(\Omega^{\prime}\right)+\left\|\delta \mu_{V^{0}}\right\|\left(\Omega^{\prime}\right)+M_{\Omega^{\prime}}\left(\partial\left[\left|V^{0}\right|\right]\right) \leq C\left(\Omega^{\prime}\right)\right\}
\end{gathered}
$$

Here, $M_{\Omega^{\prime}}(\cdot)$ denotes the mass in the sense of currents.

The Helfrich energy of $V^{0}$ is (see also [13, Eq. (2.1)])

$$
\begin{aligned}
W_{H_{0}}\left(V^{0}\right)= & \int_{M}\left|H_{V^{0}}(x)-H_{0}(* \xi(x))\right|^{2} \theta_{+}(x) \\
& +\left|H_{V^{0}}(x)+H_{0}(* \xi(x))\right|^{2} \theta_{-}(x) \mathrm{d} \mathcal{H}^{2}(x) \\
= & \int\left|H_{V^{0}}(x)-H_{0}(* \xi)\right|^{2} \mathrm{~d} V^{0}(x, \xi) .
\end{aligned}
$$

Furthermore, we need to define the enclosed volume and the area of such oriented varifolds:

$$
\operatorname{Area}\left(V^{0}\right):=\mu_{V^{0}}\left(\mathbb{R}^{3}\right), \quad \operatorname{Vol}\left(V^{0}\right):=\frac{1}{3} \int\langle x,(* \xi)\rangle \mathrm{d} V^{0}(x, \xi)
$$

Now let $\Sigma$ be a smooth oriented two-dimensional manifold and $f: \Sigma \rightarrow \mathbb{R}^{3}$ a smooth immersion. To employ the compactness criterion (2.2) we need to define a corresponding oriented integral varifold (see [13, Sect. 2]): Let $\xi_{f}: f(\Sigma) \rightarrow G^{0}(2,3)$ be an $\mathcal{H}^{2}$ measurable orientation. Then the corresponding oriented integral varifold is

$$
V_{f}^{0}:=V^{0}\left(f(\Sigma), \theta_{+}, \theta_{-}, \xi_{f}\right)
$$

To define the densities, let us denote the chosen continuous orientation of $T_{x} \Sigma$ by $\tau(x)$. Then $\theta_{+}, \theta_{-}: f(\Sigma) \rightarrow \mathbb{N}_{0}$ are defined by

$$
\begin{aligned}
& \theta_{+}(y)=\sum_{x \in f^{-1}(y)} \operatorname{sign}_{+}\left(d f(\tau(x)) /\left(\xi_{f}(y)\right),\right. \\
& \theta_{-}(y)=\sum_{x \in f^{-1}(y)} \operatorname{sign}_{-}\left(\mathrm{d} f(\tau(x)) /\left(\xi_{f}(y)\right) .\right.
\end{aligned}
$$

Here 


$$
\operatorname{sign}_{+}\left(d f(\tau(x)) /\left(\xi_{f}(y)\right)= \begin{cases}1, & \text { if } \xi_{f}(y) \text { is the same orientation as } \mathrm{d} f(\tau(x)) \\ 0, & \text { else. }\end{cases}\right.
$$

Analogously, sign_ $\left(d f(\tau(x)) /\left(\xi_{f}(y)\right)=1\right.$, if $\xi_{f}(y)$ is the opposite orientation of $d f(\tau(x))$. Please note that these densities are only well defined $\mathcal{H}^{2} \mid f(\Sigma)$ almost everywhere, which is enough to obtain a well defined oriented varifold.

Let $f_{k} \in M_{\text {Area }_{0}, \text { Vol }_{0}}$ be a minimising sequence as in Theorem 1.1 with orientation $\xi_{k}: f_{k}(\Sigma) \rightarrow G^{0}(2,3)$ and no boundary, i.e. closed. Furthermore let

$$
V_{k}^{0}:=V^{0}\left(f_{k}(\Sigma), \theta_{+}^{k}, \theta_{-}^{k}, \xi_{k}\right),
$$

with $\theta_{ \pm}^{k}$ defined as in (2.5). Let $H_{k}: f_{k}(\Sigma) \rightarrow \mathbb{R}^{3}$ be the mean curvature vector of $V_{k}^{0}$. Then the Helfrich energy of $f_{k}$ becomes

$$
W_{H_{0}}\left(f_{k}\right)=\int_{f_{k}(\Sigma)}\left|H_{k}(x)-H_{0}\left(* \xi_{k}(x)\right)\right|^{2} \theta_{+}^{k}+\left|H_{k}(x)+H_{0}\left(* \xi_{k}(x)\right)\right|^{2} \theta_{-}^{k} \mathrm{~d} \mathcal{H}^{2} .
$$

By Cauchy-Schwartz's and Young's inequality we get for $\varepsilon>0$

$$
\begin{aligned}
& \int_{f_{k}(\Sigma)}\left|H_{k}(x)-H_{0}\left(* \xi_{k}(x)\right)\right|^{2} \theta_{+}^{k} \mathrm{~d} \mathcal{H}^{2} \\
& \left.\quad=\int_{f_{k}(\Sigma)}\left(\left|H_{k}\right|^{2}-2 H_{0}\left\langle H_{k},\left(* \xi_{k}\right)\right\rangle+H_{0}^{2}\right)\right) \theta_{+}^{k} \mathrm{~d} \mathcal{H}^{2} \\
& \quad \geq \int_{f_{k}(\Sigma)}\left(\left(\left|H_{k}\right|^{2}-2\left|H_{0}\right|\left|H_{k}\right|+H_{0}^{2}\right) \theta_{+}^{k} \mathrm{~d} H^{2}\right. \\
& \geq \int_{f_{k}(\Sigma)}\left(\left|H_{k}\right|^{2}-\varepsilon\left|H_{k}\right|^{2}-\frac{1}{\varepsilon} H_{0}^{2}+H_{0}^{2}\right) \theta_{+}^{k} \mathrm{~d} \mathcal{H}^{2} \\
& =(1-\varepsilon) \int_{f_{k}(\Sigma)}\left|H_{k}\right|^{2} \theta_{+}^{k} \mathrm{~d} \mathcal{H}^{2}+\left(1-\frac{1}{\varepsilon}\right) H_{0}^{2} \int_{f_{k}(\Sigma)} \theta_{+}^{k} \mathrm{~d} \mathcal{H}^{2} .
\end{aligned}
$$

By repeating the argument with $\theta_{-}^{k}$, we obtain for $\varepsilon \in(0,1)$

$$
\frac{W_{H_{0}}\left(f_{k}\right)+\left(\frac{1}{\varepsilon}-1\right) H_{0}^{2} \text { Area }_{0}}{1-\varepsilon} \geq \int_{\Sigma_{k}}\left|H_{k}\right|^{2}\left(\theta_{+}^{k}+\theta_{-}^{k}\right) \mathrm{d} \mathcal{H}^{2}=\int\left|H_{k}\right|^{2} \mathrm{~d} \mu_{k} .
$$

Hence there is a $C=C\left(H_{0}\right)>0$, such that

$$
\int\left|H_{k}\right|^{2} \mathrm{~d} \mu_{k} \leq C \cdot\left(W_{H_{0}}\left(f_{k}\right)+\operatorname{Area}\left(f_{k}\right)\right) .
$$

By the definition of $M_{\text {Area }_{0}, \text { Vol }_{0}}$, we also have

$$
0 \in f_{k}(\Sigma)
$$

for every $k \in \mathbb{N}$. Since the $f_{k}$ are closed, we have $\partial\left[\left|V_{k}^{0}\right|\right]=0$. Hence, equation (2.6) allows us to employ Hutchinsons compactness result for oriented integral varifolds [22, Thm. 3.1] rsp. (2.2) and obtain an oriented integral 2-varifold $V^{0}=V^{0}\left[M, \theta_{+}, \theta_{-}, \xi\right]$, such that after extracting a subsequence and relabeling we have 


$$
V_{k}^{0} \rightarrow V^{0} \text { as oriented varifolds. }
$$

Furthermore let $A_{k}$ be the second fundamental form of $f_{k}$. By (2.6) and since the topology of $f_{k}$ is fixed, we get

$$
\int_{f_{k}(\Sigma)}\left|A_{k}\right|^{2} \mathrm{~d} \mathcal{H}^{2} \leq C
$$

for some constant $C>0$ independent of $k$ (see also [35, Eq. (1.1)]). Hence, by [22, Thm. 5.3.2] $\mu:=\mu_{V^{0}}$ has a weak second fundamental form $A_{\mu} \in L^{2}(\mu)$. By possibly extracting another subsequence, we obtain

$$
\begin{aligned}
V_{k}^{0} & \rightarrow V^{0} \text { as oriented varifolds, } \\
\mu_{k} & \rightarrow \mu \text { weakly as varifolds, } \\
\left|A_{k}\right|^{2} \mu & \rightarrow v \text { weakly as Radon measures, } \\
\left|A_{\mu}\right|^{2} \mu & \leq v \text { and } v\left(\mathbb{R}^{3}\right) \leq C<\infty .
\end{aligned}
$$

Without loss of generality we also have $M=\operatorname{spt}(\mu)$.

Before we can proceed, we need to ensure that the limit $V^{0}$ satisfies $\operatorname{Area}\left(V^{0}\right)=A_{0}$ and $\operatorname{Vol}\left(V^{0}\right)=\operatorname{Vol}_{0}$. For the first one, we use the following lemma

Lemma 2.1 (see [13], Lemma 2.1) $\operatorname{spt}(\mu)$ is compact.

Proof The proof is analouge to [13, Lemma 2.1]. For the reader's convenience we include it here. Simon's diameter estimate [39, Lemma 1.1] and the bound on the Willmore energy yield

$$
\operatorname{diam}\left(f_{k}(\Sigma)\right) \leq C \sqrt{\mu_{k}\left(\mathbb{R}^{3}\right) \cdot W_{\text {Will }}\left(f_{k}\right)} \leq C
$$

Now let $x \in \operatorname{spt}(\mu)$. For an arbitrary $\rho>0$ we obtain by, e.g. [27, Prop. 4.26] and the defintion of the support of a Radon measure

$$
0<(\mu)\left(B_{\rho}(x)\right) \leq \liminf _{k \rightarrow \infty}\left(\mu_{k}\right)\left(B_{\rho}(x)\right) .
$$

Hence $\operatorname{spt}\left(\mu_{k}\right) \cap B_{\rho}(x) \neq \emptyset$ for $k$ big enough. Therefore, we can find $x_{k} \in \operatorname{spt}\left(\mu_{k}\right)$ such that $x_{k} \rightarrow x$. By (2.11), we finally obtain

$$
\operatorname{diam}(\operatorname{spt}(\mu)) \leq C
$$

and the lemma is proven.

Furthermore, (2.11) and (2.9) yield a constant $N>0$, such that for all $k$ we have

$$
\operatorname{spt}(\mu), f_{k}(\Sigma) \subset B_{N}(0) .
$$

Now choose a smooth cut-off function $\varphi \in C_{0}^{\infty}\left(B_{2 N}(0)\right)$, such that $\varphi=1$ on $B_{N}(0)$. The varifold convergence of $\mu_{k}$ now yields

$$
\text { Area }_{0}=\lim _{k \rightarrow \infty} \mu_{k}\left(\mathbb{R}^{3}\right)=\lim _{k \rightarrow \infty} \int \varphi \mathrm{d} \mu_{k}=\int \varphi \mathrm{d} \mu=\mu\left(\mathbb{R}^{3}\right) .
$$


Also $(x, \xi) \mapsto \varphi(x)\langle x, *(\xi)\rangle$ defines a continuous function with compact support on $\mathbb{R}^{3} \times G^{0}(2,3)$. Hence, the oriented varifold convergence yields

$$
\begin{array}{r}
\operatorname{Vol}_{0}=\operatorname{Vol}\left(f_{k}\right)=\frac{1}{3} \int \varphi(x)\langle x, *(\xi)\rangle \mathrm{d} V_{k}^{0}(x, \xi) \\
\rightarrow \frac{1}{3} \int \varphi(x)\langle x, *(\xi)\rangle \mathrm{d} V^{0}(x, \xi)=\operatorname{Vol}\left(V^{0}\right) .
\end{array}
$$

The enclosed volume will need more attention, since we have to calculate a first variation. We will do this in Sect. 3

\section{First Variation of enclosed volume}

In this section, we derive a suitable formula for the first variation of the enclosed volume with respect to a smooth vectorfield. Let $V^{0}=V^{0}\left[M, \theta_{+}, \theta_{-}, \xi\right]$ be an oriented 2-integral varifold on $\mathbb{R}^{3}$ with compact support, finite mass and empty boundary, i.e. $\partial\left[\left|V^{0}\right|\right]=0$. Then the isoperimetric inequality for currents [38, Thm 30.1] yields an integral 3-current $R \in D_{3}\left(\mathbb{R}^{3}\right)$ with

$$
\partial R=\left[\left|V^{0}\right|\right], \quad(M(R))^{\frac{2}{3}} \leq C M\left(\left[\left|V^{0}\right|\right]\right) \leq C \mu_{V^{0}}\left(\mathbb{R}^{3}\right)<\infty
$$

for some constant $C>0$ independent of $R$ or $\left[\left|V^{0}\right|\right]$ and such that $R$ has compact support. Since $R$ is three-dimensional in $\mathbb{R}^{3},\left[38\right.$, Remark 26.28] yields a function $\theta_{R}: \mathbb{R}^{3} \rightarrow \mathbb{Z}$ of bounded variation, such that for every $\omega \in D^{3}\left(\mathbb{R}^{3}\right)$ we have

$$
R(\omega)=\int\left\langle\omega, e_{1} \wedge e_{2} \wedge e_{3}\right\rangle \theta_{R} \mathrm{~d} \mathcal{L}^{3}
$$

Now we claim the following equation, which we will prove afterwards:

$$
\operatorname{Vol}\left(V^{0}\right)=-\int \theta_{R} \mathrm{~d} \mathcal{L}^{3}
$$

As a short remark: If $V^{0}$ would be given by an embedded closed surface, $R$ would represent the open and bounded set with boundary $V^{0}$.

Since $\theta_{R}$ is of bounded variation, we find a Borel measure $\left|\nabla \theta_{R}\right|$ and a Borel measurable function $\sigma_{R}: \mathbb{R}^{3} \rightarrow \partial B_{1}(0)$, such that for every smooth $g: \mathbb{R}^{3} \rightarrow \mathbb{R}^{3}$ with compact support we have (see, e.g. [15, Section 5.1])

$$
\int \operatorname{div} g \theta_{R} \mathrm{~d} \mathcal{L}^{3}=-\int\left\langle g, \sigma_{R}\right\rangle \mathrm{d}\left|\nabla \theta_{R}\right| .
$$

We now claim

$$
\int\left\langle g, \sigma_{R}\right\rangle \mathrm{d}\left|\nabla \theta_{R}\right|=\int\langle g(x), *(\xi)\rangle \mathrm{d} V^{0}(x, \xi)
$$

for every $g \in C_{c}^{\infty}\left(\mathbb{R}^{3}, \mathbb{R}^{3}\right)$. The proof is as follows: We define $\omega:=-g_{1} d x_{2} \wedge \mathrm{d} x_{3}+g_{2} d x_{1} \wedge \mathrm{d} x_{3}-g_{3} \mathrm{~d} x_{1} \wedge \mathrm{d} x_{2}$ and then [38, Remark 26.28] yields: 


$$
\begin{aligned}
\int\left\langle g, \sigma_{R}\right\rangle \mathrm{d}\left|\nabla \theta_{R}\right| & =-\int \theta_{R} \operatorname{div}(g) \mathrm{d} \mathcal{L}^{3} \\
& =-R(d \omega)=-(\partial R)(\omega)=-\left[\left|V^{0}\right|\right](\omega) \\
& =-\int\langle\omega(x), \xi\rangle \mathrm{d} V^{0}(x, \xi) .
\end{aligned}
$$

Furthermore, we have

$$
\begin{gathered}
\xi=\xi_{1} e_{2} \wedge e_{3}+\xi_{2} e_{1} \wedge e_{3}+\xi_{3} e_{1} \wedge e_{2} \\
\Rightarrow * \xi=\xi_{1} e_{1}-\xi e_{2}+\xi e_{3} .
\end{gathered}
$$

Hence,

$$
\begin{aligned}
\langle\omega, \xi\rangle & =-g_{1} \xi_{1}+g_{2} \xi_{2}-g_{3} \xi_{3} \\
\Rightarrow\langle g, *(\xi)\rangle & =g_{1} \xi_{1}-g_{2} \xi_{2}+g_{3} \xi_{3}=-\langle\omega, \xi\rangle .
\end{aligned}
$$

which yields (3.4).

Since $R$ has compact support and by multiplying with a smooth cutoff function, (3.4) is valid for every smooth vectorfield. Hence, we can apply (3.4) to $g(x)=\frac{1}{3} x$ and obtain

$$
\begin{aligned}
\int 1 \cdot \theta_{R} \mathrm{~d} \mathcal{L}^{3} & =\frac{1}{3} \int \operatorname{div}(x) \theta_{R}(x) \mathrm{d} \mathcal{L}^{3}(x)=-\frac{1}{3} \int\left\langle x, \sigma_{R}\right\rangle \mathrm{d}\left|\nabla \theta_{R}\right| \\
& =-\frac{1}{3} \int\langle x, * \xi\rangle \mathrm{d} V^{0}(x, \xi)=-\operatorname{Vol}\left(V^{0}\right),
\end{aligned}
$$

which is (3.2).

Under our assumptions, i.e. finite mass and compact support, the current $R$ is unique. Let us prove this claim: Assume we have $R_{1}, R_{2} \in D_{3}\left(\mathbb{R}^{3}\right)$ with compact support and finite mass satisfying

$$
\partial R_{1}=\left[\left|V^{0}\right|\right], \quad \partial R_{2}=\left[\left|V^{0}\right|\right]
$$

Then

$$
\partial\left(R_{1}-R_{2}\right)=0
$$

and

$$
M\left(R_{1}-R_{2}\right) \leq M\left(R_{1}\right)+M\left(R_{2}\right)<\infty .
$$

Now the constancy theorem for currents (see, e.g. [38, Thm. 26.27]) yields a $c \in \mathbb{R}$, such that

$$
R_{1}-R_{2}=c\left[\left|\mathbb{R}^{3}\right|\right]
$$

Since $R_{1}-R_{2}$ has finite mass, $c=0$, i.e.

$$
R_{1}=R_{2} \text {. }
$$

If we want to calculate the first derivative of the enclosed volume, we need to make sense of mapping an oriented integral varifold by a diffeomorphism. So let $g: \mathbb{R}^{3} \rightarrow \mathbb{R}^{3}$ be a diffeomorphism. Then we define $g_{\sharp} V^{0}:=V^{0}\left[\tilde{M}, \tilde{\theta}_{+}, \tilde{\theta}_{-}, \tilde{\xi}\right]$ by 


$$
\begin{aligned}
\tilde{M} & :=g(M) \\
\tilde{\theta}_{ \pm}(x) & :=\theta_{ \pm}\left(g^{-1}(x)\right) \\
\tilde{\xi}(x) & :=\left(\frac{d^{M} g_{y \sharp} \xi(y)}{\left|d^{M} g_{y \sharp} \xi(y)\right|}||_{y=g^{-1}(x)} .\right.
\end{aligned}
$$

Then we have for every $\omega \in D^{2}\left(\mathbb{R}^{3}\right)$

$$
\begin{aligned}
& {\left[\left|g_{\sharp} V^{0}\right|\right](\omega)} \\
& \quad=\int_{g(M)}\left\langle\omega(x),\left.\left(\frac{d^{M} g_{y \sharp} \xi(y)}{\left|\mathrm{d}^{M} g_{y \sharp} \xi(y)\right|}\right)\right|_{y=g^{-1}(x)}\right\rangle\left(\theta_{+}\left(g^{-1}(x)\right)-\theta_{-}\left(g^{-1}(x)\right)\right) \mathrm{d} \mathcal{H}^{2}
\end{aligned}
$$

By [38, Remark 27.2] we also have

$$
\begin{aligned}
& \left(g_{\sharp}\left[\left|V^{0}\right|\right]\right)(\omega) \\
& \quad=\int_{g(M)}\left\langle\omega(x),\left.\left(\frac{d^{M} g_{y \sharp} \xi(y)}{\left|\mathrm{d}^{M} g_{y \sharp} \xi(y)\right|}\right)\right|_{y=g^{-1}(x)}\right\rangle\left(\theta_{+}\left(g^{-1}(x)\right)-\theta_{-}\left(g^{-1}(x)\right)\right) \mathrm{d} \mathcal{H}^{2}
\end{aligned}
$$

and hence

$$
g_{\sharp}\left[\left|V^{0}\right|\right]=\left[\left|g_{\sharp} V^{0}\right|\right] .
$$

Now we are ready to calculate the first variation of the enclosed volume. Let $X \in C_{c}^{\infty}\left(\mathbb{R}^{3}, \mathbb{R}^{3}\right)$ be a vectorfield and $\Phi: \mathbb{R} \times \mathbb{R}^{3} \rightarrow \mathbb{R}^{3}$ the corresponding flow, i.e. $\partial_{t}(\Phi(t, x))=X(\Phi(t, x))$ and $\Phi(0, x)=x$ for all $t, x$. Since $x \mapsto \Phi(t, x)$ is a diffeomorphism for every $t, \Phi(t, \cdot)_{\sharp}\left[\left|V^{0}\right|\right]$ is well defined. Equation (3.8) now yields with [38, Remark 26.21]

$$
\partial\left(\left[\left|\Phi(t, \cdot)_{\sharp} V^{0}\right|\right]\right)=\partial\left(\Phi(t, \cdot)_{\sharp}\left[\left|V^{0}\right|\right]\right)=\Phi(t, \cdot)_{\sharp}\left(\partial\left[\left|V^{0}\right|\right]\right)=\Phi(t, \cdot)_{\sharp} R .
$$

Hence the uniqueness property of $R$ and (3.3) give us the first equality

$$
\operatorname{Vol}\left(\Phi(t, \cdot)_{\sharp} V^{0}\right)=-\int \theta_{\Phi(t,)_{\sharp} R} \mathrm{~d} \mathcal{L}^{3}=-\int \theta_{R}\left(\Phi(t, \cdot)^{-1}(x)\right) \mathrm{d} \mathcal{L}^{3}(x) .
$$

The second equality follows from [38, Remark 27.2] and the fact, that $x \mapsto \Phi(t, x)$ is orientation preserving for every $t \in \mathbb{R}$. If we now decompose $\theta_{R}$ into positiv and negativ parts, we can employ the calculation for the first variation of varifolds (see, e.g. [38, Sect. 16]) and we finally obtain

$$
\left.\left(\frac{\partial}{\partial t} \operatorname{Vol}\left(\Phi(t, \cdot)_{\sharp} V^{0}\right)\right)\right|_{t=0}=-\int \operatorname{div}(X) \theta_{R} \mathrm{~d} \mathcal{L}^{3}=\int\left\langle X, \sigma_{R}\right\rangle \mathrm{d}\left|\nabla \theta_{R}\right| .
$$




\section{Area and volume correction}

In this chapter, we introduce a two parameter diffeomorphism of $\mathbb{R}^{3}$ to adjust an error appearing in the graphical decomposition method by Simon, see [39, Sect. 3] for the prescribed area and enclosed volume. This diffeomorphism will be constructed by two vector fields and their corresponding flows (cf. Fig. 1).

This idea was introduced by Schygulla in [36] for a one parameter diffeomorphism and prescribed isoperimetric ratio. We will expand this method by using a version of the inverse function theorem (see Theorem A.2) with some explicit bounds on the size of the set of invertibility.

Before we can start working on our diffeomorphism, we have to define currents for the minimising sequence $f_{k}$, with which we will be able to calculate the enclosed volume as in Sect. 3. So let $\left(R_{k}\right)_{k \in \mathbb{N}} \subset D_{3}\left(\mathbb{R}^{3}\right)$ be a sequence of integral currents satisfying (see also (3.1))

$$
\begin{aligned}
\partial R_{k} & =\left[\left|V_{k}^{0}\right|\right] \\
\text { and } M\left(R_{k}\right)^{\frac{2}{3}} & \leq C M\left(\left[\left|V_{k}^{0}\right|\right]\right) .
\end{aligned}
$$

Here, $C>0$ is given by the isoperimetric inequality [38, Thm. 30.1]. As in Sect. 3 there are functions of bounded variation $\theta_{R_{k}}: \mathbb{R}^{3} \rightarrow \mathbb{Z}$ representing $R_{k}$ as in (3.2). Since

$$
M\left(R_{k}\right) \leq C M\left(\partial R_{k}\right)^{\frac{3}{2}}=C M\left(\left[\left|V_{k}^{0}\right|\right]\right)^{\frac{3}{2}} \leq C \mu_{k}\left(\mathbb{R}^{3}\right)^{\frac{3}{2}}=C V o l_{0}^{\frac{3}{2}}<\infty,
$$

the compactness theorem for currents [38, Thm. 27.3] rsp. for functions of bounded variation [15, Section 5.2.3] yield a convergent subsequence of $R_{k}$ rsp. $\theta_{k}$ in the current rsp. BV sense. After relabeling, we can hence assume $R_{k} \rightarrow R$ weakly as currents and $\theta_{R_{k}} \rightarrow \theta_{R}$ in the BV sense. Here, $\theta_{R}: \mathbb{R}^{3} \rightarrow \mathbb{Z}$ satisfies (3.2) with respect to $R$. Since the boundaries of currents also converge, we have

$$
\partial R=\left[\left|V^{0}\right|\right]
$$

Since $R$ has finite mass, the uniqueness result (3.6) also yields $M(R)^{\frac{2}{3}} \leq C M\left(\left[\left|V^{0}\right|\right]\right)$.

Let us define the following sequence of functions corresponding to the area and enclosed volume: For this we need $X, Y \in C_{c}^{\infty}\left(\mathbb{R}^{3}, \mathbb{R}^{3}\right)$. Let $\Phi_{X}$ rsp. $\Phi_{Y}$ be the flow of $X$ rsp. $Y$. Now let

Fig. 1 Correcting Area and Volume outside of a prescribed region

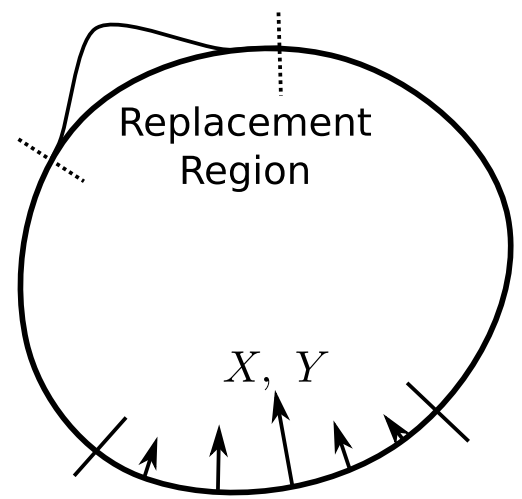




$$
\Phi(s, t, x):=\Phi_{X}\left(s, \Phi_{Y}(t, x)\right)
$$

for $s, t \in \mathbb{R}$ and $x \in \mathbb{R}^{3}$. Let

$$
F(s, t):=\left(\begin{array}{c}
\left(\Phi(s, t, \cdot)_{\sharp} \mu\right)\left(\mathbb{R}^{3}\right) \\
\operatorname{Vol}\left(\Phi(s, t, \cdot)_{\sharp} V^{0}\right)
\end{array}\right) .
$$

and

$$
F_{k}(s, t):=\left(\begin{array}{c}
\left(\Phi(s, t, \cdot)_{\sharp} \mu_{k}\right)\left(\mathbb{R}^{3}\right) \\
\operatorname{Vol}\left(\Phi(s, t, \cdot)_{\sharp} V_{k}^{0}\right)
\end{array}\right) .
$$

Since $\Phi$ does not depend on $k$, we obtain $F_{k}^{1} \rightarrow F^{1}$ pointwise as $k \rightarrow \infty$. Furthermore, $\theta_{R_{k}}$ converges in the BV-sense and we therefore have $L^{1}$-convergence $\theta_{R_{k}} \rightarrow \theta_{R}$. Hence, for all $s, t \in \mathbb{R}$

$$
\begin{gathered}
\operatorname{Vol}\left(\Phi(s, t, \cdot)_{\sharp} V_{k}^{0}\right) \stackrel{(3.10)}{=}-\int \theta_{R_{k}}\left(\Phi(s, t, \cdot)^{-1}(x)\right) \mathrm{d} \mathcal{L}^{3}(x) \\
=-\int \theta_{R_{k}}|\operatorname{det}(D \Phi)| \mathrm{d} \mathcal{L}^{3} \rightarrow-\int \theta_{R}|\operatorname{det}(D \Phi)| \mathrm{d} \mathcal{L}^{3} \\
=-\int \theta_{R}\left(\Phi^{-1}(s, t, x)\right) \mathrm{d} \mathcal{L}^{3} \stackrel{(3.10)}{=} \operatorname{Vol}\left(\Phi(s, t, \cdot)_{\sharp} V^{0}\right),
\end{gathered}
$$

which yields the pointwise convergence $F_{k} \rightarrow F$. Here ${ }^{-1}$ and $D$ refer to the $x$-variable of $\Phi$.

Since we want to locally invert $F$ and $F_{k}$, we have to derive $F$ and find $X, Y$ such that $D F(0,0)$ is invertible. Equation (3.11) and the usual calculation of the first variation of a varifold (see, e.g. [38, Sect. 16]) yields

$$
D F(0,0)=\left(\begin{array}{cc}
\int \operatorname{div}_{T_{x} \mu} X(x) \mathrm{d} \mu(x) & \int \operatorname{div}_{T_{x} \mu} Y(x) \mathrm{d} \mu(x) \\
-\int \theta_{R} \operatorname{div} X \mathrm{~d} \mathcal{L}^{3} & -\int \theta_{R} \operatorname{div} Y \mathrm{~d} \mathcal{L}^{3}
\end{array}\right)
$$

To find the necessary vectorfields, we work similarly to [36, Lemma 4] and prove the following two lemmas:

Lemma 4.1 (cf. Lemma 4 in [36]) There exists an $r>0$, such that for every $x \in \operatorname{spt}(\mu)$ there exists a point $\eta \in \operatorname{spt}(\mu) \backslash B_{r}(x)$, such that for every $\varepsilon>0$ there exists a vectorfield $Y \in C_{0}^{\infty}\left(B_{\varepsilon}(\eta), \mathbb{R}^{3}\right)$ satisfying

$$
\int \theta_{R} \operatorname{div} Y \mathrm{~d} x=-\int\left\langle Y, \sigma_{R}\right\rangle \mathrm{d}\left|\nabla \theta_{R}\right| \neq 0 .
$$

Proof We proceed by contradiction and assume the statement is false. Then there exist sequences $r_{k} \rightarrow 0$ and $x_{k} \in \operatorname{spt}(\mu)$, such that for all $\eta \in \operatorname{spt}(\mu) \backslash B_{r_{k}}\left(x_{k}\right)$ there is an $\varepsilon_{\eta}>0$ such that

$$
\int\left\langle Y, \sigma_{R}\right\rangle \mathrm{d}\left|\nabla \theta_{R}\right|=0
$$

for all $Y \in C_{0}^{\infty}\left(B_{\varepsilon_{\eta}}(\eta), \mathbb{R}^{3}\right)$. By Lemma $2.1 \operatorname{spt}(\mu)$ is compact and thus we can extract a subsequence and relabel such that $x_{k} \rightarrow x \in \operatorname{spt}(\mu)$. Hence we obtain 


$$
\sigma_{R}=0\left|\nabla \theta_{R}\right| \text {-a.e. . }
$$

Thus by (3.5)

$$
\operatorname{Vol}\left(V^{0}\right)=0,
$$

which is a contradiction to $\operatorname{Vol}\left(V^{0}\right)=\operatorname{Vol}_{0} \neq 0$.

Lemma 4.2 (cf. Lemma 4 in [36]) Either $H_{\mu} \in L^{\infty}(\mu)$ or there exists an $r>0$, such that for every $z \in \operatorname{spt}(\mu)$ there exist two points $\eta_{Y}, \eta_{X} \in \operatorname{spt}(\mu) \backslash B_{r}(z)$, such that for every $\varepsilon_{Y}, \varepsilon_{X}>0$ there exist vectorfields $Y \in C_{0}^{\infty}\left(B_{\varepsilon_{Y}}\left(\eta_{Y}\right)\right)$ and $X \in C_{0}^{\infty}\left(B_{\varepsilon_{X}}\left(\eta_{X}\right)\right)$ satisfying

$$
\begin{aligned}
& \int \operatorname{div}_{T_{x} \mu} X(x) \mathrm{d} \mu(x) \int\left\langle Y, \sigma_{R}\right\rangle \mathrm{d}\left|\nabla \theta_{R}\right| \\
& -\int \operatorname{div}_{T_{x} \mu} Y(x) \mathrm{d} \mu(x) \int\left\langle X, \sigma_{R}\right\rangle \mathrm{d}\left|\nabla \theta_{R}\right| \neq 0 .
\end{aligned}
$$

Proof Let us assume that we do not find an $r>0$ as requested. Then we find sequences $r_{k} \searrow 0, z_{k} \in \operatorname{spt}(\mu)$, such that for every $\eta_{Y}, \eta_{X} \in \operatorname{spt}(\mu) \backslash B_{r_{k}}\left(z_{k}\right)$ there are $\varepsilon_{Y, \eta_{Y}}, \varepsilon_{X, \eta_{X}}>0$, which satisfy

$$
\begin{aligned}
& \int \operatorname{div}_{T_{x} \mu} X(x) \mathrm{d} \mu(x) \int\left\langle Y, \sigma_{R}\right\rangle \mathrm{d}\left|\nabla \theta_{R}\right| \\
& -\int \operatorname{div}_{T_{x} \mu} Y(x) \mathrm{d} \mu(x) \int\left\langle X, \sigma_{R}\right\rangle \mathrm{d}\left|\nabla \theta_{R}\right|=0
\end{aligned}
$$

for every $Y \in C_{0}^{\infty}\left(B_{\varepsilon_{Y, \eta_{Y}}}\left(\eta_{Y}\right)\right)$ and $X \in C_{0}^{\infty}\left(B_{\varepsilon_{X, \eta_{X}}}\left(\eta_{X}\right)\right)$. Since $\operatorname{spt}(\mu)$ is compact, we find a convergent subsequence of $z_{k}$ and after relabeling we have $z_{k} \rightarrow z \in \operatorname{spt}(\mu)$. Hence for every $\eta_{Y}, \eta_{X} \in \operatorname{spt}(\mu) \backslash\{z\}$ there are $\varepsilon_{Y, \eta_{Y}}, \varepsilon_{X, \eta_{X}}>0$, such that every $Y \in C_{0}^{\infty}\left(B_{\varepsilon_{Y, \eta_{Y}}}\left(\eta_{Y}\right), \mathbb{R}^{3}\right)$ and $X \in C_{0}^{\infty}\left(B_{\varepsilon_{X, \eta_{X}}}\left(\eta_{X}\right), \mathbb{R}^{3}\right)$ satisfies (4.6). According to Lemma 4.1, we find an $\eta \in \operatorname{spt}(\mu) \backslash\{z\}$ and a $Y \in C_{0}^{\infty}\left(B_{\varepsilon_{Y, \eta}}(\eta)\right)$ with

$$
\int\left\langle Y, \sigma_{R}\right\rangle \mathrm{d}\left|\nabla \theta_{R}\right| \neq 0
$$

From now on this $Y$ is fixated. All in all we find for every $\eta_{X} \in \operatorname{spt}(\mu) \backslash\{z\}$ a radius $\varepsilon_{\eta_{X}}$ such that for every $X \in C_{0}^{\infty}\left(B_{\varepsilon_{X, \eta_{X}}}\right)$ we have

$$
\begin{aligned}
& \int \operatorname{div}_{T_{x} \mu} X(x) \mathrm{d} \mu(x) \int\left\langle Y, \sigma_{R}\right\rangle \mathrm{d}\left|\nabla \theta_{R}\right| \\
& -\int \operatorname{div}_{T_{x} \mu} Y(x) \mathrm{d} \mu(x) \int\left\langle X, \sigma_{R}\right\rangle \mathrm{d}\left|\nabla \theta_{R}\right|=0
\end{aligned}
$$

Hence 


$$
\begin{aligned}
\int_{\text {spt( }(\mu)}\left\langle H_{\mu}, X\right\rangle\left(\theta_{+}+\theta_{-}\right) \mathrm{d} \mathcal{H}^{2} & =-\int \operatorname{div}_{T_{x} \mu} X(x) \mathrm{d} \mu(x) \\
& =C\left(\varepsilon_{Y}\right) \int\left\langle X, \sigma_{R}\right\rangle \mathrm{d}\left|\nabla \theta_{R}\right| \\
& \stackrel{(3.4)}{=} C\left(\varepsilon_{Y}\right) \int\langle X,(* \xi)\rangle \mathrm{d} V^{0} \\
& =C\left(\varepsilon_{Y}\right) \int_{\operatorname{spt}(\mu)}\langle X(x),(* \xi(x))\rangle\left(\theta_{+}-\theta_{-}\right) \mathrm{d} \mathcal{H}^{2}(x)
\end{aligned}
$$

Since $\eta_{X}$ is arbitrary and $\mu(\{z\})=0$, we have

$$
H_{\mu}\left(\theta_{+}+\theta_{-}\right)=C\left(\varepsilon_{Y}\right) \cdot(* \xi)\left(\theta_{+}-\theta_{-}\right) \quad \mu \text {-a.e. . }
$$

Furthermore $\theta_{+}+\theta_{-} \geq 1 \mu$-a.e. and $\theta_{ \pm} \in \mathbb{N}_{0}$ finally yield $H_{\mu} \in L^{\infty}(\mu)$.

Since we like to apply Theorem A.2 to $F_{k}$, we need to be able to estimate the difference of two values of the first derivative independently of $k$. To do this, we employ the mean value theorem and hence need to estimate the second derivative:

Lemma 4.3 For every $X, Y \in C_{c}^{\infty}\left(\mathbb{R}^{3}, \mathbb{R}^{3}\right)$ and every $T>0$, there exists a constant $C=C\left(\|\Phi\|_{C^{3}\left(B_{T}(0) \times \mathbb{R}^{3}\right)}\right.$, Area $_{0}$, Vol $\left._{0}\right)>0$, such that for every $s, t \in B_{T}(0)$ we have for every $k \in \mathbb{N}$ :

$$
\left|D^{2} F_{k}(s, t)\right| \leq C
$$

Proof We start by estimating $F^{2}$ :

Since (3.10) only needs the corresponding map to be a diffeomorphism, we obtain the same result for $x \mapsto \Phi(s, t, x)$. Furthermore, the usual substitution formula yields:

$$
\begin{aligned}
F_{k}^{2}(s, t) & =-\int \theta_{R_{k}}\left(\Phi(s, t, \cdot)^{-1}(x)\right) \mathrm{d} \mathcal{L}^{3}(x) \\
& =-\int\left|\operatorname{det}\left(D_{x} \Phi(s, t, x)\right)\right| \theta_{R_{k}}(x) \mathrm{d} \mathcal{L}^{3}(x) .
\end{aligned}
$$

Now there exists a constant $C>0$ only dependend on $T>0, X, Y$ and their respective derivatives, such that for every $s, t \in(-T, T)$ we have

$$
D_{s, t}^{2}\left|\operatorname{det}\left(D_{x} \Phi(s, t, x)\right)\right|, D_{s, t}^{2}\left|\operatorname{det}\left(D_{x} \Phi(s, t, x)\right)\right| \leq C .
$$

Hence, for these $s, t$ the isoperimetric inequality [38, Thm 30.1] yields

$$
\left|D^{2} F_{k}^{2}(s, t)\right| \leq C \int\left|\theta_{R_{k}}\right| \mathrm{d} \mathcal{L}^{3} \leq C\left(\mu_{k}\left(\mathbb{R}^{3}\right)\right)^{\frac{3}{2}}=C\left(\text { Area }_{0}\right)^{\frac{3}{2}}
$$

Now let us turn to $F^{1}$ :

Since $x \mapsto \Phi(s, t, x)$ is a diffeomorphism, we can apply [38, Eq. 15.7] and obtain

$$
F_{k}^{1}(s, t)=\int\left(J_{\mu_{k}} \Phi(s, t, x)\right) \mathrm{d} \mu_{k}
$$


Furthermore, in the Jacobian $J_{\mu_{k}} \Phi(s, t, x)$ the measure $\mu_{k}$ is independent of $s$ and $t$. Also the derivatives $d^{\mu_{k}} \Phi(s, t, x)$ appearing in the Jacobian, can be estimated by the full derivative of $x \mapsto \Phi(s, t, x)$. Therefore there is a constant $C>0$ only dependend on $T>0, X, Y$ and their respective derivatives, such that for every $s, t \in(-T, T)$ we have

$$
\left|J_{\mu_{k}} \Phi(s, t, x)\right| \leq C .
$$

This yields

$$
\left|D^{2} F^{1}(s, t)\right| \leq C \mu_{k}\left(\mathbb{R}^{3}\right)=C \cdot \text { Area }_{0},
$$

which is the desired conclusion.

If we change the minimising sequence by $\Phi$, we also have to be sure, that the Helfrich energy and the second fundamental form is controlled as well:

Lemma 4.4 (cf. [36] p. 915, Eq. (v)) For every $X, Y \in C_{c}^{\infty}\left(\mathbb{R}^{3}, \mathbb{R}^{3}\right)$ and every $T>0$, there exists a constant $C=C\left(\|\Phi\|_{C^{3}\left(B_{T}(0) \times \mathbb{R}^{3}\right)}\right.$, Area $\left._{0}, \operatorname{Vol}_{0}\right)>0$ (independent of $\left.k\right)$, such that for every $s, t \in(-T, T)$ we have

$$
\left.\left|D_{s, t} \int\right| A_{k}^{s, t}\right|^{2} \mathrm{~d} \mu_{k} \mid \leq C .
$$

Here, $A_{k}^{s, t}$ is the second fundamental form of $\Sigma \ni x \mapsto \Phi\left(s, t, f_{k}(x)\right)$.

Proof By a partition of unity on $\Sigma$ and a rigid motion we can assume, that we can write $x \mapsto \Phi\left(s, t, f_{k}(x)\right)$ locally as a smooth graph with small Lipschitz norm. Hence, we have $u_{k}: \mathbb{R}^{2} \supset B_{r}(0) \rightarrow \mathbb{R}$ smooth with $\left|\nabla u_{k}\right| \leq 1$ and $u_{k}(0)=0$, which satisfies for a small open set $U \subset \Sigma$ :

$$
f_{k}(U)=\operatorname{graph}\left(u_{k}\right) .
$$

Now we can calculate the second fundamental form of $\Phi\left(s, t, f_{k}(\cdot)\right)$ by using $u_{k}$ and chain rule:

$$
\begin{aligned}
\partial_{i}\left(\Phi\left(s, t,\left(x, u_{k}(x)\right)\right)\right)= & \sum_{j=1}^{2}\left(\partial_{j} \Phi\right)\left(s, t,\left(x, u_{k}(x)\right)\right) \delta_{i j} \\
& +\left(\partial_{3} \Phi\right)\left(s, t,\left(x, u_{k}(x)\right)\right) \partial_{i} u_{k}(x) \\
= & \left(\partial_{i} \Phi\right)\left(s, t,\left(x, u_{k}(x)\right)\right)+\left(\partial_{3} \Phi\right)\left(s, t,\left(x, u_{k}(x)\right)\right) \partial_{i} u_{k}(x) .
\end{aligned}
$$

Then the second derivatives are as follows: 


$$
\begin{aligned}
\partial_{\ell} \partial_{i}\left(\Phi\left(s, t,\left(x, u_{k}(x)\right)\right)\right)= & \sum_{m=1}^{2}\left(\partial_{m} \partial_{i} \Phi\right)\left(s, t,\left(x, u_{k}(x)\right) \delta_{m \ell}\right. \\
& +\left(\partial_{3} \partial_{i} \Phi\right)\left(s, t,\left(x, u_{k}(x)\right) \partial_{\ell} u_{k}(x)\right. \\
& +\sum_{m=1}^{2}\left(\partial_{m} \partial_{3} \Phi\right)\left(s, t,\left(x, u_{k}(x)\right)\right) \delta_{m \ell} \partial_{i} u_{k}(x) \\
& +\left(\partial_{3}^{2} \Phi\right)\left(s, t,\left(x, u_{k}(x)\right)\right) \partial_{\ell} u_{k}(x) \partial_{i} u_{k}(x) \\
& +\partial_{3} \Phi\left(s, t,\left(x, u_{k}(x)\right)\right) \partial_{\ell} \partial_{i} u_{k}(x) .
\end{aligned}
$$

The unit normal can be expressed by

$$
n(s, t, x)= \pm \frac{\partial_{1}\left(\Phi\left(s, t,\left(x, u_{k}(x)\right)\right)\right) \times \partial_{2}\left(\Phi\left(s, t,\left(x, u_{k}(x)\right)\right)\right)}{\left|\partial_{1}\left(\Phi\left(s, t,\left(x, u_{k}(x)\right)\right)\right) \times \partial_{2}\left(\Phi\left(s, t,\left(x, u_{k}(x)\right)\right)\right)\right|}
$$

Since $x \mapsto \Phi(s, t, x)$ is a diffeomorphism of $\mathbb{R}^{3}$, such that it is the identity outside of a ball, we find a constant $C=C(T)>0$, such that for every $(s, t) \in B_{T}(0)$ we have

$$
\left|\operatorname{det} D_{x} \Phi(s, t, x)\right| \geq C .
$$

This yields a noncollapsing of the basis of the tangential space, i.e.

$$
\left|\partial_{1}\left(\Phi\left(s, t,\left(x, u_{k}(x)\right)\right)\right) \times \partial_{2}\left(\Phi\left(s, t,\left(x, u_{k}(x)\right)\right)\right)\right| \geq C>0
$$

for a possibly different constant $C=C(T)>0$. The second fundamental form can be expressed in these coordinates as

$$
\left(A_{k}^{s, t}\right)_{i \ell}=\left\langle\partial_{\ell} \partial_{i}\left(\Phi\left(s, t,\left(x, u_{k}(x)\right)\right)\right), n(s, t, x)\right\rangle .
$$

All in all we therefore have

$$
D_{s, t}\left(\left|A_{k}^{s, t}\right|^{2}\right) \leq C\left(1+\left\|\nabla u_{k}\right\|_{L^{\infty}}^{4}+\left\|D^{2} u_{k}\right\|_{L^{\infty}}^{2}\right)
$$

with some constant $C>0$ independent of $k$. Since we imposed a small Lipschitz Norm on $u_{k}$ we deduce by, e.g. [9, p. 5]

$$
D_{s, t}\left(\left|A_{k}^{s, t}\right|^{2}\right) \leq C\left(1+\left|A_{k}\right|^{2}\right)
$$

Hence

$$
\left.\left|D_{s, t} \int\right| A_{k}^{s, t}\right|^{2} \mathrm{~d} \mu_{k} \mid \leq C
$$

and $C$ does not depend on $k$.

Remark 4.5 With the same techniques as in Lemma 4.4 we can also show (cf. (4.8))

$$
\left|D_{s, t} W_{H_{0}}\left(f_{k}^{s, t}\right)+D_{s, t} \operatorname{Area}\left(f_{k}^{s, t}\right)\right| \leq C
$$

for all $(s, t) \in B_{T}(0)$ and $C$ is independent of $k$. Here $f_{k}^{s, t}$ is the immersion $\Sigma \ni x \mapsto \Phi\left(s, t, f_{k}(x)\right)$. 


\section{Partial regularity and lower semicontinuity}

In this section, we adapt the partial regularity method introduced by Simon (see [39, Section 3]). This method is based on replacing parts of the minimising sequence with biharmonic graphs and compare the resulting energies. Here we use the idea of Schygulla [36, Lemma 5] to correct the area and enclosed volume of the modified sequence, so that they become competitors for the minimum of the Helfrich energy again.

Let $\varepsilon_{0}>0$ be fixated. In dependence of this $\varepsilon_{0}$ we say $x_{0} \in \operatorname{spt}(\mu)$ is a good point, iff (see $(2.10))$

$$
\nu\left(\left\{x_{0}\right\}\right)<\varepsilon_{0}^{2} .
$$

In neighbourhoods of these good points we will show $C^{1, \beta}$ regularity and a graphical decomposition of $\mu$. Since we work with immersions with possible self intersections, we also use the ideas of Schätzle (see [35, Prop. 2.2]) to implement Simon's regularity method (cf. [39, Section 3]). The lemma is now as follows (cf. also [13, Lemma 3.1]):

Lemma 5.1 For any $\varepsilon>0$ there exist $\varepsilon_{0}=\varepsilon_{0}\left(H_{0}\right.$, Vol $_{0}$, Area $\left._{0}, \varepsilon\right)>0$, $\theta=\theta\left(H_{0}\right.$, Vol $_{0}$, Area $\left._{0}, \varepsilon\right)>0, \quad \rho_{0}=\rho_{0}\left(H_{0}\right.$, Vol $_{0}$, Area $\left._{0}, \varepsilon\right)>0 \quad$ and $\beta=\beta\left(H_{0}\right.$, Vol $_{0}$, Area $\left._{0}\right)>0$, such that for every good point $x_{0} \in \operatorname{spt}(\mu)$ and good radius $0<\rho_{x_{0}} \leq \rho_{0}$ satisfying

$$
v\left(\overline{B_{\rho_{x_{0}}}\left(x_{0}\right)}\right)<\varepsilon_{0}^{2},
$$

$\mu \quad$ is a union of $\left(W^{2,2} \cap C^{1, \beta}\right)$-graphs in $B_{\theta \rho_{x_{0}}}\left(x_{0}\right)$ of functions $u_{i} \in\left(W^{2,2} \cap C^{1, \beta}\right)\left(B_{\theta \rho_{x_{0}}}\left(x_{0}\right) \cap L_{i}\right)$. Here, $L_{i} \subset \mathbb{R}^{3}$ are two-dimensional affine spaces and $i=1, \ldots, I_{x_{0}} \leq C\left(\right.$ Area $_{0}$, Vol $\left._{0}, H_{0}\right)$. Furthermore, the $u_{i}$ satisfy the following estimate

$$
\begin{aligned}
& \left(\theta \rho_{x_{0}}\right)^{-1}\left\|u_{i}\right\|_{L^{\infty}\left(B_{\theta \rho_{x_{0}}}\left(x_{0}\right) \cap L_{i}\right)} \\
& \quad+\left\|\nabla u_{i}\right\|_{L^{\infty}\left(B_{\theta \rho_{x_{0}}}\left(x_{0}\right) \cap L_{i}\right)}+\left(\theta \rho_{x_{0}}\right)^{\beta} h \ddot{o} l_{B_{\theta \rho_{x_{0}}}\left(x_{0}\right) \cap L_{i}, \beta}\left(\nabla u_{i}\right) \leq \varepsilon .
\end{aligned}
$$

Moreover, we have a power-decay for the second fundamental form, i.e. $\forall x \in \frac{B_{\frac{\theta \rho_{x_{0}}}{4}}}{{ }^{4}}\left(x_{0}\right)$, $0<\rho<\frac{\theta \rho_{x_{0}}}{4}$

$$
\int_{B_{\rho}(x)}\left|A_{\mu}\right|^{2} \mathrm{~d}(\mu) \leq C\left(H_{0}, \text { Area }_{0}, \text { Vol }_{0}\right)\left(\varepsilon_{0}^{2}+\rho_{0}\right) \rho^{\beta} \rho_{x_{0}}^{-\beta} .
$$

Proof We start by applying the graphical decomposition lemma of Simon (see [39, Lemma 2.1]) to the minimising sequence $f_{k}: \Sigma \rightarrow \mathbb{R}^{3}$. This lemma is also applicable for immersions by an argument of Schätzle (see [35, p. 280] and cf. [13, Lemma A.6], in the beginning we work as in these papers). We repeat some steps of [35, (2.11)-(2.16)], which we will need later (see also [13, (3.5)-(3.14)]):

The upper-semicontinuity of the weak convergence $\left|A_{k}\right|^{2} \mu_{k} \rightarrow v$ for Radon measures (see, e.g. [27, Prop. 4.26]) yields for every ball satisfying (5.1)

$$
\limsup _{k \rightarrow \infty} \int_{B_{\rho_{x_{0}}}\left(x_{0}\right)}\left|A_{k}\right|^{2} \mathrm{~d} \mu_{k} \leq v\left(\overline{B_{\rho_{x_{0}}}\left(x_{0}\right)}\right)<\varepsilon_{0}^{2} .
$$


Hence, the aforementioned graphical decomposition lemma [13, Lemma A.6] is applicable and we can decompose $f_{k}^{-1}\left(\overline{B_{\frac{\rho_{0}}{2}}\left(x_{0}\right)}\right)$ for large $k$ and $\varepsilon_{0}>0$ small enough into closed pairwise disjoint sets $D_{k, i} \subset \Sigma$ (which are topological discs), $i=1, \ldots, I_{k}, I_{k} \leq C E_{H_{0}, \text { Area }_{0}, \text { Vol }_{0}}$ (cf. (2.7), (1.6)), i.e.

$$
f_{k}^{-1}\left(\overline{B_{\frac{\rho_{0}}{2}}\left(x_{0}\right)}\right)=\bigcup_{i=1}^{I_{k}} D_{k, i} .
$$

Furthermore, for every $i=1, \ldots, I_{k}$ we have affine two-dimensional planes $L_{k, i} \subset \mathbb{R}^{3}$, smooth functions $u_{k, i}: \bar{\Omega}_{k, i} \subset L_{k, i} \rightarrow L_{k, i}^{\perp}\left(\Omega_{k, i}=\Omega_{k, i}^{0} \backslash \cup_{m} d_{k, i, m}, \Omega_{k, i}^{0} \subset L_{k, i}\right.$ simply connected, $d_{k, i, m}$ closed pairwise disjoint discs), satisfying

$$
\rho_{x_{0}}^{-1}\left|u_{k, i}\right|+\left|\nabla u_{k, i}\right| \leq C\left(E_{H_{0}, \text { Area }_{0}, \text { Vol }_{0}}\right) \varepsilon_{0}^{\frac{1}{22}}
$$

and pairwise disjoint topological discs $P_{k, i, 1}, \ldots, P_{k, i, J_{k}} \subset D_{k, i}$, such that

$$
f_{k}\left(D_{k, i}-\bigcup_{j=1}^{J_{k, i}} P_{k, i, j}\right)=\operatorname{graph}\left(u_{k, i}\right) \cap \overline{B_{\frac{\rho_{x_{0}}}{2}}\left(x_{0}\right)}
$$

and

$$
\sum_{i=1}^{I_{k}} \sum_{j=1}^{J_{k}} \operatorname{diam} f_{k}\left(P_{k, i, j}\right) \leq C\left(E_{H_{0}, \text { Area }_{0}, \text { Vol }_{0}}\right) \varepsilon_{0}^{\frac{1}{2}} \rho_{x_{0}} .
$$

The arguments [35, Eq. (2.12)-(2.14)] yield for a chosen $0<\tau<\frac{1}{2}$ an $\varepsilon_{0}$ small enough and $0<\theta<\frac{1}{4}$, such that

$$
\frac{\mu_{g_{k}}\left(D_{k, i} \cap f_{k}^{-1}\left(B_{\sigma}(x)\right)\right)}{w_{2} \sigma^{2}}<1+\tau
$$

for $B_{\sigma}(x) \subset B_{\theta \rho_{x_{0}}}\left(x_{0}\right)$ arbitrary. Here, $w_{2}$ denotes the Hausdorff measure of the two-dimensional euclidean unit ball and $\mu_{g_{k}}$ the area measure on $\Sigma$ induced by $f_{k}$. As in [35, p. 281] rsp. [13, Eq. (3.8)] we define Radon measures on $\mathbb{R}^{3}$, which will lead to a decomposition of $\mu$ by Radon measures of Hausdorff densitity one:

$$
\begin{aligned}
\mu_{k, i} & :=\mathcal{H}^{2}\left\lfloor f_{k}\left(D_{k, i} \cap f_{k}^{-1}\left(B_{\theta \rho_{x_{0}}}\left(x_{0}\right)\right)\right)\right. \\
& =f_{k}\left(\mu_{g_{k}}\left\lfloor\left(D_{k, i} \cap f_{k}^{-1}\left(B_{\theta \rho_{x_{0}}}\left(x_{0}\right)\right)\right)\right) .\right.
\end{aligned}
$$

Since the $\mu_{k, i}$ are integer rectifiable, they are of density one, by (5.8). Furthermore we have as in [35, p. 281] rsp. [13, Eq. (3.9)]

$$
\sum_{i=1}^{I_{k}} \mu_{k, i}=\mu_{k}\left\lfloor B_{\theta \rho_{x_{0}}}\left(x_{0}\right)\right.
$$

After taking a subsequence depending on $x_{0}, \theta \rho_{x_{0}}$ and relabeling we can assume $I_{k}=I$ and

$$
\mu_{k, i} \rightarrow \mu_{i} \text { weakly as varifolds in } B_{\theta \rho_{x_{0}}}\left(x_{0}\right) .
$$


As shown in [13, Eq. (3.13)] (see also [35, p. 281]) we get

$$
\mu\left\lfloor B_{\theta \rho_{x_{0}}}\left(x_{0}\right)=\sum_{i=1}^{I} \mu_{i} .\right.
$$

As in [35, Eq. (2.16)] we can pass to the limit in (5.8) and obtain

$$
\frac{\mu_{i}\left(B_{\sigma}(x)\right)}{w_{2} \sigma^{2}} \leq 1+\tau \quad \forall \overline{B_{\sigma}(x)} \subset B_{\theta \rho_{x_{0}}}\left(x_{0}\right) .
$$

We will apply Allard's regularity theorem A.1 to the $\mu_{i}$. Inequality (5.13) already takes care of the needed density estimate (A.3). The remainder of the proof will be about showing (A.2) or a similar $L^{p}$-bound for the mean curvature. Here we need to make a distinction of cases given by Lemma 4.2.

First we assume $H_{\mu} \in L^{\infty}$ :

In this case, we will show that $H_{\mu_{i}} \in L^{p}, p>2$ arbitrary. By the usual regularity Theorem of Allard (see, e.g. [38, Thm. 24.2]), the $\mu_{i}$ will be $C^{1, \beta}$ graphs.

We need the definition of the tilt and height excess (see, e.g. [33, Eqs. (1.1),(1.2)]):

$$
\begin{gathered}
\operatorname{tiltex}_{\mu}(x, \omega, T):=\omega^{-2} \int_{B_{\omega}(x)}\left\|T_{\xi} \mu-T\right\|^{2} \mathrm{~d} \mu(\xi), \\
\operatorname{heightex}_{\mu}(x, \omega, T):=\omega^{-4} \int_{B_{\omega}(x)} \operatorname{dist}(\xi-x, T)^{2} \mathrm{~d} \mu(\xi) .
\end{gathered}
$$

Here $x \in \mathbb{R}^{3}, \omega \in \mathbb{R}$ and $T \subset \mathbb{R}^{3}$ is a two-dimensional subspace (cf. [38, Sect. 38] for defining a norm on subspaces, i.e. the unoriented Grassmannian). Since $H_{\mu} \in L^{\infty}(\mu)$, [33, Thm. 5.1] yields

$$
\text { tiltex }_{\mu}(x, \omega, T), \operatorname{heightex}_{\mu}(x, \omega, T) \leq O_{x}\left(\omega^{2}\right) .
$$

By the defintion of the height and tilt excess we obtain for every $i=1, \ldots, I$

$$
\text { tiltex }_{\mu_{i}}(x, \omega, T) \leq \operatorname{tiltex}_{\mu}(x, \omega, T) \leq O_{x}\left(\omega^{2}\right)
$$

and

$$
\text { heightex }_{\mu_{i}}(x, \omega, T) \leq \text { heightex }_{\mu}(x, \omega, T) \leq O_{x}\left(\omega^{2}\right),
$$

because $\mu_{i} \leq \mu$. Furthermore by [34, Thm. 3.1] $\mu_{i}$ and $\mu$ are $C^{2}$-rectifiable. Hence [34, Cor. $4.3]$ is applicable and yields

$$
H_{\mu_{i}}=H_{\mu}, \quad \mu_{i} \text {-a.e } .
$$

Since $H_{\mu} \in L^{\infty}(\mu)$ and $\mu_{i} \leq \mu$ we finally obtain

$$
H_{\mu_{i}} \in L^{\infty}\left(\mu_{i}\right)
$$

which yields by the usual Allard regularity theorem (see, e.g. [38, Thm. 24.2]), that every $\mu_{i}$ is a $C^{1, \beta}$-graph with $\beta \in(0,1)$ arbitrary.

This case is therefore done. 
Now we modify Schygulla's argument [36, Lemma 5] to our situation: The beginning of the argument is as in [39, Lemma 3.1]. For the readers convenience and because we need the notation, we repeat these steps here (see also [13, pp. 9-10]):

Let us choose $0<\rho<\theta \rho_{x_{0}}$ fixed but arbitrary. We need to apply the graphical decomposition Lemma [39, Lemma 2.1] again to $f_{k}\left(D_{k, i}\right) \cap \overline{B_{\rho}\left(x_{0}\right)}$. Hence we obtain smooth functions $v_{k, i, \ell}: \tilde{\Omega}_{k, i, \ell} \subset \tilde{L}_{k, i, \ell} \rightarrow \tilde{L}_{k, i, \ell}^{\perp}, \tilde{L}_{k, i} \subset \mathbb{R}^{3}$ two-dimensional planes $\left(\ell=1, \ldots, N_{k, i} \leq C\left(E_{H_{0}, \text { Area }_{0}, \text { Vol }_{0}}\right)\right), \quad \tilde{\Omega}_{k, i}=\tilde{\Omega}_{k, i}^{0} \backslash \cup_{m} \tilde{d}_{k, i, \ell, m}, \quad \tilde{\Omega}_{k, i}^{0}$ simply connected and $\tilde{d}_{k, i, \ell, m}$ closed pairwise disjoint discs. Furthermore we have

$$
\rho^{-1}\left|v_{k, i, \ell}\right|+\left|\nabla v_{k, i, \ell}\right| \leq C\left(E_{H_{0}, \text { Area }_{0}, \text { Vol }_{0}}\right) \varepsilon_{0}^{\frac{1}{22}}
$$

and closed pairwise disjoint topological discs $\tilde{P}_{k, i, \ell, 1}, \ldots, \tilde{P}_{k, i, \ell, J_{k, i, \ell}} \subset \tilde{D}_{k, i, \ell}\left(\tilde{D}_{k, i, \ell}\right.$ is a topological disc as well) such that for all $\ell$

$$
f_{k}\left(\tilde{D}_{k, i, \ell}-\bigcup_{j=1}^{J_{k, i, \ell}} \tilde{P}_{k, i, \ell, j}\right) \cap \overline{B_{\rho}\left(x_{0}\right)}=\operatorname{graph}\left(v_{k, i, \ell}\right) \cap \overline{B_{\rho}\left(x_{0}\right)}
$$

These $\tilde{P}_{k, i, \ell, j}$ also satisfy the following estimate

$$
\sum_{j=1}^{J_{k, i, \ell}} \operatorname{diam} f_{k}\left(\tilde{P}_{k, i, \ell, j}\right) \leq C\left(E_{H_{0}, \text { Area }_{0}, \text { Vol }_{0}}\right) \varepsilon_{0}^{\frac{1}{2}} \rho \leq \frac{1}{8} \rho,
$$

if we choose $C\left(E_{H_{0}, \text { Area }_{0}, \text { Vol }_{0}}\right) \varepsilon_{0}^{\frac{1}{2}}<\frac{1}{8}$ (The need for (5.21) is also the reason for applying the graphical decomposition a second time). Let us also introduce the corresponding Radon measures similar to (5.9)

$$
\tilde{\mu}_{k, i, \ell}:=\mathcal{H}^{2}\left\lfloor f_{k}\left(\tilde{D}_{k, i, \ell}\right)=\mu_{k, i}\left\lfloor f_{k}\left(\tilde{D}_{k, i, \ell}\right) .\right.\right.
$$

Since $\left.f_{k}\right|_{D_{k, i} \cap f_{k}^{-1}\left(B_{\theta \rho_{x_{0}}}\left(x_{0}\right)\right)}$ is an embedding, we also have

$$
\sum_{\ell=1}^{N_{k, i}} \tilde{\mu}_{k, i, \ell}=\mu_{k, i} \mid B_{\rho}\left(x_{0}\right) \text {. }
$$

Let us define

$$
C_{\sigma}^{k, i, \ell}\left(x_{0}\right):=\left\{x+y: x \in B_{\sigma}\left(x_{0}\right) \cap \tilde{L}_{k, i, \ell}, y \in \tilde{L}_{k, i, \ell}^{\perp}\right\} .
$$

Inequality (5.21) yields $\mathcal{L}^{1}$-measurable sets $S_{k} \subset\left(\frac{1}{2} \rho, \frac{3}{4} \rho\right)$, such that $\forall j=1, \ldots, J_{k, i, \ell}$

$$
\mathcal{L}^{1}\left(S_{k}\right) \geq \frac{1}{8} \rho \text { and } \forall \sigma \in S_{k}: \partial C_{\sigma}^{k, i, \ell}\left(x_{0}\right) \cap f\left(\tilde{P}_{k, i, \ell, j}\right)=\emptyset .
$$

Therefore $\left.v_{k, i, \ell}\right|_{\partial B_{\sigma}\left(x_{0}\right) \cap \tilde{L}_{k, i, \ell}}$ and $\left.\nabla v_{k, i, \ell}\right|_{\partial B_{\sigma}\left(x_{0}\right) \cap \tilde{L}_{k, \ell, \ell}}$ are well defined for any $\sigma \in S_{k}$. Hence Lemma A.3 is applicable and yields a function $w_{k, i, \ell}: B_{\sigma}\left(x_{0}\right) \cap \tilde{L}_{k, i, \ell} \rightarrow \tilde{L}_{k, i, \ell}^{\perp}$ with Dirichlet boundary data given by $v_{k, i, \ell}$ and $\nabla v_{k, i, \ell}$.

This defines a sequence of immersions $f_{k, \sigma}^{\text {graph }}: \Sigma_{k, \sigma} \rightarrow \mathbb{R}^{3}$ by

$$
\Sigma_{k, \sigma}:=\left(\Sigma \backslash\left(f_{k}^{-1}\left(B_{\sigma}\left(x_{0}\right)\right) \cap \tilde{D}_{k, i}\right)\right) \oplus\left(B_{\sigma}\left(x_{0}\right) \cap \tilde{L}_{m, i}\right)
$$

and 


$$
f_{k, \sigma}^{g r a p h}(p)=\left\{\begin{array}{c}
f_{k}(p), \quad p \in \Sigma \backslash\left(f_{k}^{-1}\left(B_{\sigma}\left(x_{0}\right)\right) \cap \tilde{D}_{k, i}\right) \\
w_{k, i, \ell}(p), \quad p \in\left(B_{\sigma}\left(x_{0}\right) \cap \tilde{L}_{m, i}\right) .
\end{array}\right.
$$

Since $\tilde{D}_{k, i}$ is topologically a disc, $\Sigma$ and $\Sigma_{k, \sigma}$ are topologically equivalent. $f_{k, \sigma}^{\text {graph }}$ does not have the same area or enclosed volume as $f_{k}$, which we like to correct now. Therefore we need some estimates on these properties. We start with the area. Here we use (5.8) and Lemma A.3 to obtain

$$
\begin{aligned}
\left|\operatorname{Area}\left(f_{k}\right)-\operatorname{Area}\left(f_{k, \sigma}^{g r a p h}\right)\right| & \leq 2 \max \left\{\operatorname{Area}\left(\left.f_{k}\right|_{\tilde{D}_{k, i}}\right), \operatorname{Area}\left(w_{k, i, \ell}\right)\right\} \\
& \leq 2 \max \left\{\tilde{\mu}_{k, i, \ell}\left(B_{\sigma}\left(x_{0}\right)\right), C \int_{B_{\sigma}\left(x_{0}\right) \tilde{L}_{k, i, \ell}} \mathrm{d} \mathcal{H}^{2}\right\} \\
& \leq C \sigma^{2} \leq C \rho^{2} .
\end{aligned}
$$

Let us proceed with the enclosed volume. Since $f_{k}(\Sigma) \subset B_{N}(0)$, for $N>0$ big enough and independent of $k$, we also get $f_{k, \sigma}^{\text {graph }}\left(\Sigma_{k, \sigma}\right) \subset B_{N}(0)$. Hence the definition of the enclosed volume and the Cauchy-Schwartz inequality yield

$$
\begin{aligned}
\left|\operatorname{Vol}\left(f_{k}\right)-\operatorname{Vol}\left(f_{k, \sigma}^{g r a p h}\right)\right| & \leq 2 \max \left\{\left|\operatorname{Vol}\left(\left.f_{k}\right|_{\tilde{D}_{k, i}}\right)\right|,\left|\operatorname{Vol}\left(w_{k, i, \ell}\right)\right|\right\} \\
& \leq C(N) \max \left\{\operatorname{Area}\left(\left.f_{k}\right|_{\tilde{D}_{k, i}}\right), \operatorname{Area}\left(w_{k, i, \ell}\right)\right\} \\
& \leq C \sigma^{2} \leq C \rho^{2} .
\end{aligned}
$$

Now we apply Lemma 4.2: Hence, we find an $r>0$ and points $\eta_{X}, \eta_{Y} \in \operatorname{spt}(\mu) \backslash B_{r}\left(x_{0}\right)$ (without loss of generality we assume $\rho_{0}, \rho_{x_{0}} \leq \frac{r}{2}$ ), which satisfy that for every $\varepsilon_{X}, \varepsilon_{Y}>0$, we find vectorfields $X \in C_{0}^{\infty}\left(B_{\varepsilon_{X}}\left(\eta_{X}\right)\right)$ and $Y \in C_{0}^{\infty}\left(B_{\varepsilon_{Y}}\left(\eta_{Y}\right)\right)$, such that

$$
\begin{aligned}
& \int \operatorname{div}_{T_{x} \mu} X(x) \mathrm{d} \mu(x) \int\left\langle Y, \sigma_{R}\right\rangle \mathrm{d}\left|\nabla \theta_{R}\right| \\
& -\int \operatorname{div}_{T_{x} \mu} Y(x) \mathrm{d} \mu(x) \int\left\langle X, \sigma_{R}\right\rangle \mathrm{d}\left|\nabla \theta_{R}\right| \neq 0 .
\end{aligned}
$$

Here $R$ denotes the 3-current defined in the beginning of Sect. 4 and $\theta_{R}$ the corresponding BV-function. We also fix $\varepsilon_{X}=\varepsilon_{Y}=\rho$. Furthermore, let $\Phi$ be defined as in (4.2) and $F$ and $F_{k}$ be as in (4.3) but with respect to $f_{k, \sigma}^{g r a p h}$ instead. The results of Sect. 4 are still valid, since the diffeomorphism $\Phi$ does not influence the graphical comparison function (cf. Fig. 1). As in the beginning of Sect. 4 we obtain

$$
F_{k}(s, t) \rightarrow F(s, t) .
$$

The oriented varifold convergence and the $L^{1}$ convergence of $\theta_{R_{k}}$ yield

$$
\left|\operatorname{det} D F_{k}(0,0)\right| \rightarrow|\operatorname{det} D F(0,0)| \geq c_{0}>0
$$

for a fixed constant $c_{0}>0$. Hence, for $k$ big enough, we get

$$
\left|\operatorname{det} D F_{k}(0,0)\right| \geq \frac{c_{0}}{2} \text {. }
$$

Hence, $D F_{k}(0,0)$ is invertible. Furthermore, the mean value theorem and Lemma 4.3 yield for every $T_{0}>0$ a $C=C\left(T_{0}\right)>0$, such that for every $(s, t),\left(s^{\prime}, t^{\prime}\right) \in \overline{B_{T}(0)}$ we have 


$$
\left|D F_{k}(s, t)-D F_{k}\left(s^{\prime}, t^{\prime}\right)\right| \leq C\left\|D^{2} F_{k}\right\|_{L^{\infty}\left(\overline{B_{T}(0)}\right)}\left|(s, t)-\left(s^{\prime}, t^{\prime}\right)\right| \leq C T
$$

if we choose $0<T<T_{0}$ arbitrary. By the formula for the inverse matrix via the adjunct matrix we obtain for $k$ big enough

$$
\left|\left(D F_{k}(0,0)\right)^{-1}\right| \leq C .
$$

The constant $C$ is independent of $k$. Next we will apply Lemma A.2. Hence, we need to define a functions $\tilde{F}$ and $\tilde{F}_{k}$ satisfying the assumptions of that Lemma:

$$
\begin{aligned}
\tilde{F}(s, t) & :=(D F(0,0))^{-1}(F(s, t)-F(0,0)) \\
\tilde{F}_{k}(s, t) & :=\left(D F_{k}(0,0)\right)^{-1}\left(F_{k}(s, t)-F_{k}(0,0)\right)
\end{aligned}
$$

Here $^{-1}$ is meant as the matrix inverse. Hence

$$
\begin{array}{cc}
\tilde{F}(0,0)=0, & D \tilde{F}(0,0)=I \in \mathbb{R}^{2 \times 2}, \\
\tilde{F}_{k}(0,0)=0, & D \tilde{F}_{k}(0,0)=I \in \mathbb{R}^{2 \times 2} .
\end{array}
$$

Let furthermore $(s, t),\left(s^{\prime}, t^{\prime}\right) \in B_{T}(0)$ for $0<T<T_{0}$. Then by (5.30) and (5.31) we have

$$
\begin{aligned}
\left|D \tilde{F}_{k}(s, t)-D \tilde{F}_{k}\left(s^{\prime}, t^{\prime}\right)\right| & \leq\left|\left(D F_{k}(0,0)\right)^{-1}\right| \cdot\left|D F_{k}(s, t)-D F_{k}\left(s^{\prime}, t^{\prime}\right)\right| \\
& \leq C T<C T_{0}=: \delta_{0}<1,
\end{aligned}
$$

if we choose $T_{0}$ small enough. So Lemma A.2 is applicable to $\tilde{F}_{k}$ and therefore we find for every $(\tilde{y}, \tilde{z}) \in B_{\left(1-\delta_{0}\right) T}(0)$ parameters $\left(s_{k}, t_{k}\right)$ with

$$
\tilde{F}_{k}\left(s_{k}, t_{k}\right)=(\tilde{y}, \tilde{z}) .
$$

By (5.32) we obtain

$$
F_{k}\left(s_{k}, t_{k}\right)=F_{k}(0,0)+D F_{k}(0,0)(\tilde{y}, \tilde{z})=:(y, z) .
$$

Since $D F_{k}(0,0)$ is invertible, we obtain a $\gamma>0$ (by (5.29) only dependend on $T_{0}$ ), such that for every $(y, z) \in B_{\gamma}\left(F_{k}(0,0)\right)$ we find $\left(s_{k}, t_{k}\right) \in B_{\left(1-\delta_{0}\right) T}(0)$ satisfying

$$
F_{k}\left(s_{k}, t_{k}\right)=(y, z) \text {. }
$$

Furthermore we may choose $\gamma>0$ to be maximal, i.e. satisfying the following property: There is a $\left(y_{0}, z_{0}\right) \in \partial B_{\gamma}(0)$ and a $\left(\tilde{y}_{0}, \tilde{z}_{0}\right) \in \partial B_{\left(1-\delta_{0}\right) T}(0)$ with

$$
D F_{k}(0,0)\left(\tilde{y}_{0}, \tilde{z}_{0}\right)=\left(y_{0}, z_{0}\right) .
$$

This yields

$$
\left(1-\delta_{0}\right) T=\left|\left(\tilde{y}_{0}, \tilde{z}_{0}\right)\right|=\left|\left(D F_{k}(0,0)\right)^{-1}\left(y_{0}, z_{0}\right)\right| \leq\left|D F_{k}(0,0)^{-1}\right|\left|\left(y_{0}, z_{0}\right)\right| \leq C \gamma .
$$

Hence

$$
C\left(1-\delta_{0}\right) T \leq \gamma
$$

Now we choose $T_{0}:=\rho_{0}, T:=\rho$ (by choosing $\rho_{0}$ small enough our results are still true). For later purposes we also state that the inverse inequality of (5.34) is true as well, only with a bigger constant of course, i.e. we have with $C_{1}<C_{2}$ independent of $k$ 


$$
C_{1} \rho \leq \gamma \leq C_{2} \rho
$$

Hence $\gamma$ can at most decay linearly in $\rho$, while (5.25) and (5.26) show a quadratic error in $\rho$ for the area and volume. By choosing $\rho_{0}$ small enough, we therefore obtain for $k$ big enough parameters $\left(s_{k}, t_{k}\right) \in B_{\gamma}\left(F_{k}(0,0)\right)$ with

$$
F_{k}\left(s_{k}, t_{k}\right)=\left(\operatorname{Area}_{0}, \operatorname{Vol}_{0}\right) .
$$

Let $V_{k}^{g r a p h}$ be the oriented varifold induced by $f_{k, \sigma}^{g r a p h}$ and let us call $V_{k}^{s, t}:=\Phi(s, t, \cdot)_{\sharp} V_{k}^{g r a p h}$. The corresponding mass is called $\mu_{k}^{s, t}$. We denote with $A_{k}^{s, t}$ the second fundamentalform and with $H_{k}^{s, t}$ the mean curvature vector of $V_{k}^{s, t}$. The orientation is called $\xi_{k}^{s, t}$.

Since we cannot replace $v_{k, i, \ell}$ by $w_{k, i, \ell}$ and still have a minimising sequence, we will have to correct the resulting error by the diffeomorphism $\Phi$. By the mean value theorem and Lemma 4.4 the $L^{2}$-norm of the second fundamental form of the $\Phi$-corrected varifolds are controlled:

$$
\left|\int\right| A_{k}^{s, t}\left|\mathrm{~d} \mu_{k}^{s, t}-\int\right| A_{k}^{0,0}\left|\mathrm{~d} \mu_{k}^{s, t}\right| \leq C|(s, t)| \leq C \rho .
$$

Since $F_{k}$ is continuously differentiable and the derivative is bounded independently of $k$ (see the proof of Lemma 4.3), the area is controlled as well:

$$
\left|\mu_{k}^{s, t}\left(\mathbb{R}^{3}\right)-\mu_{k}^{0,0}\left(\mathbb{R}^{3}\right)\right|=\left|F_{k}(s, t)-F_{k}(0,0)\right| \leq C|(s, t)| \leq C \rho .
$$

The Helfrich energy is controlled by Remark 4.5 and again the mean value theorem

$$
\begin{aligned}
& \left|\int_{\operatorname{spt}(\Phi) \times G^{0}(2,3)}\right| H_{k}^{s, t}(x)-\left.H_{0}(* \xi)\right|^{2} d V_{k}^{s, t}(x, \xi) \\
& \quad-\int_{\operatorname{spt}(\Phi) \times G^{0}(2,3)}\left|H_{k}^{0,0}(x)-H_{0}(* \xi)\right|^{2} d V_{k}^{0,0}(x, \xi) \mid \leq C \rho .
\end{aligned}
$$

Let us denote with $A_{k, i, \ell}^{w}$ the second fundamental form, $H_{k, i, \ell}^{w}$ the mean curvature vector, $\xi_{w_{k, i, \ell}}$ the orientation and with $K_{k, i, \ell}^{w}$ the Gauss curvature of graph $\left(w_{k, i, \ell}\right)$. By the Gauss-Bonnet Theorem $\int_{\Sigma} K_{k} \mathrm{~d} \mu_{g_{k}}$ is given entirely by the topology of $\Sigma$. Hence $f_{k}$ is also a minimising sequence for $f \mapsto W_{H_{0}, \lambda}(f)+\kappa \int_{\Sigma} K_{f} \mathrm{~d} \mu_{g}, \kappa \in \mathbb{R}$ arbitrary under prescribed area and enclosed volume. Here $K_{f}$ denotes the Gauss curvature of a given immersion $f: \Sigma \rightarrow \mathbb{R}^{3}$. By [9, Eq. (11)] we have $\left|A_{k}\right|^{2}=\left|H_{k}\right|^{2}-2 K_{k}$ and $K_{k}$ is the Gauss curvature of $f_{k}$. The following calculation is an adaptation to our situation from [13, pp. 10-11]: 


$$
\begin{aligned}
& \int_{B_{\sigma}\left(x_{0}\right)}\left|A_{k}\right|^{2} d \tilde{\mu}_{k, i, \ell} \\
& =\int_{B_{\sigma}\left(x_{0}\right)}\left|H_{k}\right|^{2} d \tilde{\mu}_{k, i, \ell}-2 \int_{B_{\sigma}\left(x_{0}\right)} K_{k} d \tilde{\mu}_{k, i, \ell} \\
& \stackrel{(2.8)}{\leq} C\left(\int_{B_{\sigma}\left(x_{0}\right)}\left|H_{k}-H_{0}\left(* \xi_{f_{k}}\right)\right|^{2} d \tilde{\mu}_{k, i, \ell}+\tilde{\mu}_{k, i, \ell}\left(B_{\sigma}\left(x_{0}\right)\right)\right) \\
& -2 \int_{B_{\sigma}\left(x_{0}\right)} K_{k} d \tilde{\mu}_{k, i, \ell} \\
& \leq C\left(\int_{B_{\sigma}\left(x_{0}\right)}\left|H_{k}-H_{0}\left(* \xi_{f_{k}}\right)\right|^{2} d \tilde{\mu}_{k, i, \ell}-\frac{2}{C} \int_{B_{\sigma}\left(x_{0}\right)} K_{k} d \tilde{\mu}_{k, i, \ell}\right) \\
& +C \tilde{\mu}_{k, i, \ell}\left(B_{\sigma}\left(x_{0}\right)\right) \\
& \leq C\left(\int_{\operatorname{graph}\left(w_{k, i, \ell}\right)}\left|H_{k, i, \ell}^{w}-H_{0}\left(* \xi_{w_{k, i, \ell}}\right)\right|^{2} \mathrm{~d} \mathcal{H}^{2}-\frac{2}{C} \int_{\operatorname{graph}\left(w_{k, i, \ell}\right)} K_{k, i, \ell}^{w} \mathrm{~d} \mathcal{H}^{2}\right) \\
& +\left|\int_{\operatorname{spt}(\Phi) \times G^{0}(2,3)}\right| H_{k}^{s_{k}, t_{k}}-\left.H_{0}(* \xi)\right|^{2} \mathrm{~d} V_{k}^{s_{k}, t_{k}}-\frac{2}{C} \int_{\operatorname{spt}(\Phi)} K_{k}^{s_{k}, t_{k}} d \mu_{k}^{s_{k}, t_{k}} \\
& -\int_{\operatorname{spt}(\Phi) \times G^{0}(2,3)}\left|H_{k}^{0,0}-H_{0}(* \xi)\right|^{2} \mathrm{~d} V_{k}^{0,0}+\frac{2}{C} \int_{\operatorname{spt}(\Phi)} K_{k}^{0,0} d \mu_{k}^{0,0} \mid \\
& +C \tilde{\mu}_{k, i, \ell}\left(B_{\rho}\left(x_{0}\right)\right)+\varepsilon_{k} \\
& \leq C\left(\int_{\operatorname{graph}\left(w_{k, i, \ell}\right)}\left|H_{k, i, \ell}^{w}\right|^{2} \mathrm{~d} \mathcal{H}^{2}+\mathcal{H}^{2}\left(\operatorname{graph}\left(w_{k, i, \ell}\right)\right)\right. \\
& \left.+\frac{2}{C} \int_{\operatorname{graph}\left(w_{k, i, \ell}\right)} K_{k, i, \ell}^{w} \mathrm{~d} \mathcal{H}^{2}\right)+C \tilde{\mu}_{k, i, \ell}\left(B_{\rho}\left(x_{0}\right)\right)+C \rho+\varepsilon_{k} \\
& \stackrel{A .3}{\leq} C\left(\int_{B_{\sigma}\left(x_{0}\right) \cap \tilde{L}_{k, i, \ell}}\left|D^{2} w_{k, i, \ell}\right|^{2} \mathrm{~d} x+\sigma\right)+C \tilde{\mu}_{k, i, \ell}\left(B_{\rho}\left(x_{0}\right)\right)+C \rho+\varepsilon_{k} \\
& \stackrel{A .3,(5.8)}{\leq} C \sigma \int_{\operatorname{graph}\left(\left.v_{k, i, \ell}\right|_{\partial B_{\sigma}\left(x_{0}\right) n \tilde{L}_{k, i, \ell}}\right)}\left|A_{m, i}\right|^{2} \mathrm{~d} \mathcal{H}^{1}+C \sigma+C \rho+\varepsilon_{k}
\end{aligned}
$$

Here $\varepsilon_{k} \rightarrow 0$ for $k \rightarrow \infty$. The estimates connecting $D^{2} w_{k, i, \ell}$ with the corresponding curvatures can be seen by, e.g. [9, Subsection 2.1] and the bound on the gradient of $w_{k, i, \ell}$. Integrating over $S_{\rho}$ together with the Co-Area formula (see, e.g. [38, Eq. (10.6)]) yields

$$
\int_{B_{\frac{\rho}{2}}\left(x_{0}\right)}\left|A_{k}\right|^{2} \mathrm{~d} \tilde{\mu}_{k, i, \ell} \leq C \int_{B_{\frac{3}{4} \rho}\left(x_{0}\right) \backslash B_{\frac{\rho}{2}}\left(x_{0}\right)}\left|A_{k}\right|^{2} \mathrm{~d} \tilde{\mu}_{k, i, \ell}+C \rho+\varepsilon_{k} .
$$

Summing over $\ell$ yields with the help of (5.22)

$$
\int_{B_{\frac{\rho}{2}}\left(x_{0}\right)}\left|A_{k}\right|^{2} \mathrm{~d} \mu_{k, i} \leq C \int_{B_{\frac{3}{4} \rho}\left(x_{0}\right) \backslash B_{\frac{\rho}{2}}\left(x_{0}\right)}\left|A_{k}\right|^{2} \mathrm{~d} \mu_{k, i}+C \rho+\varepsilon_{k} .
$$

Hole filling yields a $0<\Theta<1$ independent of $k$, satisfying 


$$
\int_{B_{\frac{\rho}{2}}\left(x_{0}\right)}\left|A_{k}\right|^{2} d \mu_{k, i} \leq \Theta \int_{B_{\frac{3}{4} \rho}\left(x_{0}\right)}\left|A_{k}\right|^{2} \mathrm{~d} \mu_{k, i}+C \rho+\varepsilon_{k}
$$

The semi-continuity properties of $v$ (see, e.g. [27, Prop. 4.26]) for measure convergence yield for $k \rightarrow \infty$

$$
v_{i}\left(B_{\frac{\rho}{2}}\left(x_{0}\right)\right) \leq \Theta v_{i}\left(B_{\rho}\left(x_{0}\right)\right)+C \rho .
$$

Here $\left|A_{k}\right|^{2} \mu_{k, i} \rightarrow v_{i}$ for $k \rightarrow \infty$ as Radon measures. By $\mu_{k, i} \leq \mu_{k}$ we also get $v_{i} \leq \nu$. Since we only needed the estimate

$$
v\left(\overline{\left.B_{\rho_{x_{0}}}\left(x_{0}\right)\right)} \leq \varepsilon_{0}^{2}\right.
$$

to obtain (5.38), we can repeat the argument for every $B_{\rho}(x) \subset B_{\frac{\rho_{0}}{4}}\left(x_{0}\right)$. This yields for these balls

$$
v_{i}\left(B_{\frac{\rho}{2}}\left(x_{0}\right)\right) \leq \Theta v_{i}\left(B_{\rho}(x)\right)+C \rho .
$$

For example, [17, Lemma 8.23] gives us

$$
v_{i}\left(B_{\rho}(x)\right) \leq C\left(\frac{\rho}{\rho_{x_{0}}}\right)^{\beta} v_{i}\left(B_{\frac{\rho_{x_{0}}}{4}}(x)\right)+C \sqrt{\rho \rho_{x_{0}}} .
$$

Here $C=C(\Theta)>0$ and $\beta=\beta(\Theta)>0$ are constants. Since $B \frac{\rho_{x_{0}}}{4}(x) \subset B_{\rho_{x_{0}}}\left(x_{0}\right)$ we also get

$$
v_{i}\left(B_{\rho}(x)\right) \leq C\left(\frac{\rho}{\rho_{x_{0}}}\right)^{\beta} v_{i}\left(B_{\rho_{x_{0}}}\left(x_{0}\right)\right)+C \sqrt{\rho \rho_{x_{0}}} .
$$

Since $\frac{\rho}{\rho_{x_{0}}}<1$ we can choose $\beta<\frac{1}{2}$ and obtain

$$
\begin{aligned}
v_{i}\left(B_{\rho}(x)\right) & \leq C \rho^{\beta} \rho_{x_{0}}^{-\beta}\left(v_{i}\left(B_{\rho_{x_{0}}}\left(x_{0}\right)\right)+\rho^{\frac{1}{2}-\beta} \rho_{x_{0}}^{\frac{1}{2}+\beta}\right) \\
& \leq C \rho^{\beta} \rho_{x_{0}}^{-\beta}\left(v\left(\overline{B_{\rho_{0}}\left(x_{0}\right)}\right)+\rho_{0}\right) \\
& \leq C \rho^{\beta} \rho_{x_{0}}^{-\beta}\left(\varepsilon_{0}^{2}+\rho_{0}\right) .
\end{aligned}
$$

By $\left|A_{\mu_{i}}\right|^{2} \mu_{i} \leq v_{i}$ we therefore get

$$
\int_{B_{\rho}(x)}\left|A_{\mu_{i}}\right|^{2} \mathrm{~d} \mu_{i} \leq C \rho^{\beta} \rho_{x_{0}}^{-\beta}\left(\varepsilon_{0}^{2}+\rho_{0}\right) .
$$

Choosing $C\left(\varepsilon_{0}+\rho_{0}\right)$ small enough, Allard's regularity theorem A.1 (cf. (A.2)) yields $\mu_{i}$ to be a $C^{1, \beta}$ graph. By (5.12) $\mu$ is a union of $C^{1, \beta}$ graphs in a neighbourhood of $x_{0}$, which all satisfy estimates in the form of (A.5). This finishes the proof.

Remark 5.2 The proof of Lemma 5.1 does not work, if we would minimise in the class of embeddings, since the lack of a Li-Yau-type inequality prevents us from showing that the $f_{k, \sigma}^{g r a p h}$ are still embeddings. This is a key problem, because we cannot compare the Helfrich energy of $f_{k, \sigma}^{g r a p h}$ to $f_{k}$ without it. 
Next we formulate the lower-semicontinuity property of the minimising sequence:

Lemma 5.3 The minimising sequence $V_{k}^{0}$ for the Helfrich problem (1.6) satisfies

$$
W_{H_{0}}\left(V^{0}\right) \leq \liminf _{k \rightarrow \infty} W_{H_{0}}\left(V_{k}^{0}\right) .
$$

Proof Since $\mu$ is locally a graph of $C^{1, \beta} \cap W^{2,2}$ graphs outside of finitely many points (see Lemma 5.1), and these graphs are approximated by $\mu_{k, i}$, see (5.11), the proof of the lowersemicontinuity estimate is the same as in [13, Lemma 4.1].

Acknowledgements Open Access funding provided by Projekt DEAL.

Open Access This article is licensed under a Creative Commons Attribution 4.0 International License, which permits use, sharing, adaptation, distribution and reproduction in any medium or format, as long as you give appropriate credit to the original author(s) and the source, provide a link to the Creative Commons licence, and indicate if changes were made. The images or other third party material in this article are included in the article's Creative Commons licence, unless indicated otherwise in a credit line to the material. If material is not included in the article's Creative Commons licence and your intended use is not permitted by statutory regulation or exceeds the permitted use, you will need to obtain permission directly from the copyright holder. To view a copy of this licence, visit http://creativecommons.org/licenses/by/4.0/.

\section{Auxilliary results}

For the readers convenience we collect a few needed results:

The following is a variant of Allard's regularity Theorem. A proof of this statement can be found in [39, Section 3] or [37, Korollar 20.3] (see also [35, Theorem B.1]).

Theorem A.1 (Allard's regularity Theorem, see [1], Theorem 8.16) For $n, m \in \mathbb{N}$, $0<\beta<1, \alpha>0$ there exist $\varepsilon_{0}=\varepsilon_{0}(n, m, \alpha, \beta)>0, \gamma=\gamma(n, m, \alpha, \beta)$ and $C=C(n, m, \alpha, \beta)$ such that:

Let $\mu$ be an integral $n$-varifold in $B_{\rho_{0}}^{n+m}(0), 0<\rho_{0}<\infty, 0<\varepsilon<\varepsilon_{0}$ with locally bounded first variation in $B_{\rho_{0}}^{n+m}(0)$ satisfying

$$
\rho^{1-n}\|\delta \mu\|\left(B_{\rho}\right) \leq \varepsilon^{2}\left(\rho^{-n} \mu\left(B_{\rho}\right)\right)^{1-\alpha} \rho^{2 \beta} \rho_{0}^{-2 \beta}, \quad \forall B_{\rho} \subset B_{\rho_{0}}(0)
$$

or weak mean curvature $H_{\mu} \in L^{2}\left(\mu\left\lfloor B_{\rho_{0}}^{n+m}(0)\right)\right.$ satisfying

$$
\left(\rho^{2-n}\right)\left(\int_{B_{\rho}}\left|H_{\mu}\right|^{2} \mathrm{~d} \mu\right)^{\frac{1}{2}} \leq \varepsilon\left(\rho^{-n} \mu\left(B_{\rho}\right)\right)^{\frac{1}{2}-\alpha} \rho^{\beta} \rho_{0}^{-\beta}, \quad \forall B_{\rho} \subset B_{\rho_{0}}(0)
$$

and

$$
0 \in \operatorname{spt} \mu, \rho_{0}^{-n} \mu\left(B_{\rho_{0}}(0)\right) \leq(1+\varepsilon) \omega_{n} .
$$

Then there exists $u \in C^{1, \beta}\left(B_{\gamma \varepsilon \rho_{0}}^{n}(0), \mathbb{R}^{m}\right), u(0)=0$, such that after rotation

$$
\mu\left\lfloor B_{\gamma \varepsilon \rho_{0}}^{n+m}(0)=\mathcal{H}^{n}\left\lfloor\left(\operatorname{graph} u \cap B_{\gamma \varepsilon \rho_{0}}^{n+m}(0)\right)\right.\right.
$$


and

$$
\left(\varepsilon \rho_{0}\right)^{-1}\|u\|_{L^{\infty}\left(B_{\gamma \varepsilon \rho_{0}}^{n}(0)\right)}+\|\nabla u\|_{L^{\infty}\left(B_{\gamma \varepsilon \rho_{0}}^{n}(0)\right)}+\left(\varepsilon \rho_{0}\right)^{\beta} \operatorname{höl}_{B_{\gamma \varepsilon \rho_{0}}^{n}(0), \beta} \nabla u \leq C \varepsilon^{\frac{1}{2(n+1)}} .
$$

In Sect. 3 we need a version of the inverse function theorem with explicit estimates on the size of domain and codomain on which the function is invertible:

Theorem A.2 (See [26], Chapter XIV Sect. 1, Lemma 1.3) Let $0 \in U \subset \mathbb{R}^{n}$ be open and $f \in C^{1}\left(U, \mathbb{R}^{n}\right)$. Furthermore let $f(0)=0, D f(0)=i d$. Assume $r>0$ with $\overline{B_{r}(0)} \subset U$ and let $0<s<1$ satisfy

$$
\|D f(z)-D f(x)\| \leq s
$$

for all $x, z \in \overline{B_{r}(0)}$. Here $\|D f(z)\|=\sup _{|x|=1}|D f(z) x|$. If $y \in \mathbb{R}^{n}$ and $|y| \leq(1-s) r$, then there exists a unique $x \in \overline{B_{r}(0)}$, such that $f(x)=y$.

The following lemma provides a suitable comparison function in Sect. 4. It is a generalisation by Schygulla of the biharmonic comparison principle by Simon (see [39, Lemma 2.2]):

Lemma A.3 (See [36], p. 938 Lemma 8) Let $L \subset \mathbb{R}^{3}$ be a two-dimensional plane, $x_{0} \in L$ and $u \in C^{\infty}\left(U, L^{\perp}\right)$, where $U \subset L$ is an open neighbourhood of $L \cap \partial B_{\rho}\left(x_{0}\right)$. Moreover let $|D u| \leq c$ on $u$. Then there exists a function $w \in C^{\infty}\left(\overline{B_{\rho}\left(x_{0}\right)}, L^{\perp}\right)$ such that

$$
\begin{aligned}
w & =u, \frac{\partial w}{\partial \nu}=\frac{\partial u}{\partial \nu} \text { on } \partial B_{\rho}\left(x_{0}\right), \\
\frac{1}{\rho}\|w\|_{L^{\infty}\left(B_{\rho}\left(x_{0}\right)\right)} & \leq c\left(\frac{1}{\rho}\|u\|_{L^{\infty}\left(\partial B_{\rho}\left(x_{0}\right)\right)}+\|D u\|_{L^{\infty}\left(\partial B_{\rho}\left(x_{0}\right)\right)}\right), \\
\|D w\|_{L^{\infty}\left(B_{\rho}\left(x_{0}\right)\right)} & \leq c\|D u\|_{L^{\infty}\left(\partial B_{\rho}\left(x_{0}\right)\right)}, \\
\int_{B_{\rho}\left(x_{0}\right)}\left|D^{2} w(x)\right|^{2} d x & \leq c \rho \int_{\operatorname{graph} u_{\partial B_{\rho}\left(x_{0}\right)}}|A|^{2} \mathrm{~d} \mathcal{H}^{1} .
\end{aligned}
$$

Here A denotes the second fundamental form of graph $u$. Furthmore $v$ is the outer normal of $L \cap B_{\rho}\left(x_{0}\right)$ with respect to $L$.

\section{References}

1. Allard, W.K.: On the first variation of a varifold. Ann. Math. 95, 417-491 (1972)

2. Bauer, M., Kuwert, E.: Existence of minimizing Willmore surfaces of prescribed genus. Int. Math. Res. Notices 10, 553-576 (2003)

3. Canham, P.B.: The minimum energy of bending as a possible explanation of the biconcave shape of the human red blood cell. J. Theor. Biol. 26(1), 61-76 (1970)

4. Choksi, R., Morandotti, M., Veneroni, M.: Global minimizers for axisymmetric multiphase membranes. ESAIM COCV 19(4), 1014-1029 (2013)

5. Choksi, R., Veneroni, M.: Global minimizers for the doubly-constrained Helfrich energy: the axisymmetric case. Calc. Var. Partial. Differ. Equ. 48(3), 337-366 (2013)

6. Da Lio, F., Palmurella, F., Rivière, T.: A Resolution of the Poisson Problem for Elastic Plates. arXiv :1807.09373 [math.DG], 2018. Preprint 
7. Dall'Acqua, A., Deckelnick, K., Grunau, H.-C.: Classical solutions to the Dirichlet problem for Willmore surfaces of revolution. Adv. Calc. Var. 1, 379-397 (2008)

8. De Matteis, G., Manno, G.: Lie algebra symmetry analysis of the Helfrich and Willmore surface shape equations. Commun. Pur. Appl. Anal. 13(1), 453-481 (2014)

9. Deckelnick, K., Grunau, H.-C., Röger, M.: Minimising a relaxed Willmore functional for graphs subject to boundary conditions. Interfaces Free Bound. 19, 109-140 (2017)

10. Delladio, S.: Special generalized Gauss graphs and their application to minimization of functionals involving curvatures. J. Reine Angew. Math 486, 17-43 (1997)

11. Deuling, H.J., Helfrich, W.: The curvature elasticity of fluid membranes: a catalogue of vesicle shapes. J. Physique 37(11), 1335-1345 (1976)

12. Doemeland, M.: Verallgemeinerung eines Existenzsatzes für axialsymmetrische Minimierer des Willmore-Funktionals auf das Helfrich-Funktional. Scientific project, Otto-von-Guericke University Magdeburg, (2015). Supervisors: H.-Chr. Grunau, J. Wiersig

13. Eichmann, S.: The Helfrich Boundary Value Problem. Calc. Var. 58, 34 (2019). https://doi. org/10.1007/s00526-018-1468-x

14. Eichmann, S., Grunau, H.-C.: Existence for Willmore surfaces of revolution satisfying non-symmetric Dirichlet boundary conditions. Adv. Calc. Var. (2017). https://doi.org/10.1515/acv-2016-0038

15. Evans, L.C., Gariepy, R.F.: Measure Theory and fine Properties of Functions. CRC Press Inc, New York (1992)

16. Fourcade, B., Miao, L., Rao, M., Wortis, M., Zia, R.K.P.: Scaling analysis of narrow necks in curvature models of fluid lipid-bilayer vesicles. Phys. Rev. E 49(6), 5276-5287 (1994)

17. Gilbarg, D., Trudinger, N.S.: Elliptic Partial Differential Equations of Second Order, 3rd edn. Springer, Berlin (1998)

18. Große-Brauckmann, K.: New surfaces of constant mean curvature. Math. Z. 214, 527-565 (1993)

19. Grunau, H.-C.: Boundary Value Problems for the Willmore Functional. In: Proceedings of the workshop "Analysis of Shapes of Solutions to Partial Differential Equations" (2018). http://www-ian.math. uni-magdeburg.de/home/grunau/papers/Grunau_RIMS.pdf

20. Helfrich, W.: Elastic properties of lipid bilayers: Theory and possible experiments. Z. Naturforsch. C 28, 693-703 (1973)

21. Heller, L., Pedit, F.: Towards a constrained Willmore conjecture. arXiv:1705.03217v1 [math.DG], (2017). Preprint

22. Hutchinson, J.E.: Second fundamental form for varifolds and the existence of surfaces minimising curvature. Indiana U. Math. J. 35, 45-71 (1986)

23. Keller, L.G.A., Mondino, A., Rivière, T.: Embedded surfaces of arbitrary genus minimizing the Willmore energy under isoperimetric constraint. Arch. Ration. Mech. Anal. 212, 645-682 (2014)

24. Kohsaka, Y., Nagasawa, T.: On the existence for the Helfrich flow and its center manifold near spheres. Calc. Var. 16, 62-111 (2002)

25. Lamm, T., Metzger, J.: Minimizers of the Willmore functional with a small area constraint. Ann. I. H. Poincaré 30, 497-518 (2013)

26. Lang, S.: Real and Functional Analysis, 3rd edn. Springer, New York (1993)

27. Maggi, F.: Sets of Finite Perimeter and Geometric Variational Problems, 1st edn. Cambridge University Press, Cambridge (2012)

28. Marques, F.C., Neves, A.: Min-Max theory and the Willmore conjecture. Ann. Math. 149, 683-782 (2014)

29. Mondino, A., Scharrer, C.: Existence and Regularity of Spheres Minimising the Canham-Helfrich Energy. arXiv:1904.12074 [math.DG] (2019). Preprint

30. Mondino, A., Schygulla, J.: Existence of immersed spheres minimizing curvature functionals in noncompact 3-manifolds. Ann. I. H. Poincaré 31, 707-724 (2014)

31. Ou-Yang, Z., Helfrich, W.: Bending energy of vesicle membranes: General expressions for the first, second, and third variation of the shape energy and applications to spheres and cylinders. Phys. Rev. A 39(10), 5280-5288 (1989)

32. Pozetta, M.: On the Plateau-Douglas problem for the Willmore energy of surfaces with planar boundary curves. (2019). Preprint arXiv: 1810.07662

33. Schätzle, R.: Quadratic tilt-excess decay and strong maximum principle for varifolds. Ann. Scuola Norm. Sup. Pisa Cl. Sci. III(5), 171-231 (2004)

34. Schätzle, R.: Lower semicontinuity of the Willmore functional for currents. J. Differ. Geom. 81(2), 437-456 (2009)

35. Schätzle, R.: The Willmore boundary problem. Calc. Var. Partial. Differ. Equ. 37, 275-302 (2010)

36. Schygulla, J.: Willmore Minimizers with Prescribed Isoperimetric Ratio. Arch. Rational Mech. Anal. 203(3), 901-941 (2012) 
37. Schätzle, R.: Geometrische Maßtheorie. Lecture Notes, Tübingen University, (2008/2009)

38. Simon, L.: Lectures on Geometric Measure Theory. In: Proceedings of the Centre For Mathematical Analysis, Australian National University, 1st edn (1983)

39. Simon, L.: Existence of surfaces minimizing the Willmore functional. Commun. Anal. Geom. 1, 281326 (1993)

40. Thomsen, G.: Über Konforme Geometrie I: Grundlagen der konformen Flächentheorie. Hamb. Math. Abh. 3, 31-56 (1924)

41. Willmore, T.J.: Note on embedded surfaces. An. Ştiinţ. Univ. Al. I. Cuza Iaşi Seçt. I a Mat 11, $493-496$ (1965)

Publisher's Note Springer Nature remains neutral with regard to jurisdictional claims in published maps and institutional affiliations. 